\title{
Finite Element Method for Epitaxial Growth with Attachment-Detachment Kinetics
}

\author{
Eberhard Bänsch, Frank Haußer, Omar Lakkisł; Bo Li;
}

December 17, 2003

\begin{abstract}
An adaptive finite element method is developed for a class of free or moving boundary problems modeling island dynamics in epitaxial growth. Such problems consist of an adatom (adsorbed atom) diffusion equation on terraces of different height; boundary conditions on terrace boundaries including the kinetic asymmetry in the adatom attachment and detachment; and the normal velocity law for the motion of such boundaries determined by a two-sided flux, together with the one-dimensional "surface" diffusion. The problem is solved using two independent meshes: a two-dimensional mesh for the adatom diffusion and a one-dimensional mesh for the boundary evolution. The diffusion equation is discretized by the first-order implicit scheme in time and the linear finite element method in space. A technique of extension is used to avoid the complexity in the spatial discretization near boundaries. All the elements are marked, and the marking is updated in each time step, to trace the terrace height. The evolution of the terrace boundaries includes both the mean curvature flow and the surface diffusion. Its governing equation is solved by a semi-implicit front-tracking method using parametric finite elements. Simple adaptive techniques are employed in solving the adatom diffusion as well as the boundary motion problem. Numerical tests on pure geometrical motion, mass balance, and the stability of a growing circular island demonstrate that the method is stable, efficient, and accurate enough to simulate the growing of epitaxial islands over a sufficiently long time period.
\end{abstract}

*Numerical Mathematics and Scientific Computing, WIAS, Mohrenstraße 39, 10117 Berlin, Germany. Email: baensch@wias-berlin.de

${ }^{\dagger}$ Crystal Growth Group, Research Center caesar, Ludwig-Erhard-Allee 2, D-53175, Bonn, Germany. E-mail: hausser@caesar.de

${ }_{\ddagger}^{\ddagger}$ Institute of Applied and Computational Mathematics, FORTH, P.O.Box 1527, Vassilika Vouton, GR-71110, Iraklion, Greece. E-mail: omar@iacm.forth.gr

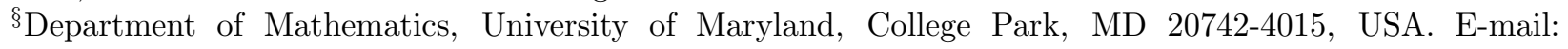
bli@math.umd.edu

${ }^{\top}$ Crystal Growth Group, Research Center caesar, Ludwig-Erhard-Allee 2, D-53175, Bonn, Germany. E-mail: voigt@caesar.de 
Keywords: epitaxial growth, island dynamics, free or moving boundary problem, adatom diffusion, attachment-detachment kinetics, surface diffusion, mean curvature flow, finite elements, adaptivity, front tracking.

AMS Mathematics Subject Classification: 35Q99, 35R35, 65N30, 65Z05, 74S05.

\section{Introduction}

We develop an adaptive finite element method for a class of free or moving boundary problems that model the island dynamics in epitaxial growth of thin films.

Epitaxial growth is a technology for growing single crystal thin films by depositing atoms and molecules onto an existing substrate. Ideally, such technique can produce high quality materials for device applications. Microscopic processes in epitaxial growth include the deposition of atoms or molecules, atom adsorption and desorption, adatom (adsorbed atom) diffusion, adatom island nucleation, the attachment and detachment of adatoms to and from island boundaries or terrace steps, and island coalescence [4, 17, 28, 40].

There are various kinds of models for epitaxial growth of thin films that are distinguished by different scales in time and space. Among them, continuum models can describe film surface morphology, predict long time growth laws in terms of scaling, and determine thermodynamic variables. One class of continuum models are the BurtonCabrera-Frank (BCF) type island dynamics models, cf. [6] and [7, 9, 15, 22, 28]. Such a model is essentially a free or moving boundary problem that consists of a diffusion equation for the adatom density on islands or terraces, boundary conditions for the moving terrace boundaries, and a velocity law for the motion of such boundaries. This moving boundary problem has the following distinguished features: First, terraces have different heights. Thus, the description of the growth is continuous in the lateral directions but discrete in the growth direction; Second, the adatom flux to the terrace boundary is two sided, from both upper and lower terraces; And third, the normal velocity of the moving terrace or island boundaries is determined by the attachment-detachment kinetics and can include one-dimensional "surface" diffusion of edge-adatoms - atoms that are diffusing along terrace boundaries.

We consider the attachment-detachment kinetics in the boundary condition for terrace boundaries that includes the Ehrlich-Schwoebel effect. In a typical step-flow or layerby-layer epitaxial growth of thin films, adatoms diffuse on a terrace and likely hit a terrace boundary. In order to stick to the boundary from an upper terrace, an adatom must overcome a higher energy barrier - the Ehrlich-Schwoebel barrier [13, 36, 37]. This asymmetry in attachment and detachment of adatoms to and from terrace boundaries has many important consequences: It induces an uphill current which in general destabilizes nominal surfaces (high symmetry surfaces) [13, 36, 37], but stabilizes vicinal surfaces (surfaces that are in the vicinity of high symmetry surfaces) with large slope, preventing step bunching [41]; It also leads to the Bales-Zangwill morphological instability of atomic steps [1, 31]; Finally, it contributes to the kinetic roughening of film surfaces [20, 29, 41].

Caflisch et al [7] have recently developed a class of island dynamics models based on step edge (terrace boundary) kinetics that involve not only the step edges or terrace boundaries and the adatom density but also the density of edge-adatoms and the density 
of kinks along terrace boundaries. Based on such kinetic models, Caflisch and Li [9] have derived a set of boundary conditions for the adatom density that includes line tension and attachment-detachment kinetics, and a normal velocity law that includes the onedimensional "surface" diffusion, cf. Figure 1. Various parts of these boundary conditions and the velocity formula have been recently suggested and partially derived based on thermodynamics $[18,22,27,28,30]$. In this work, we use these boundary conditions and normal velocity law, modified to include the convection terms in the flux, the EhrlichSchwoebel effect, and the one-dimensional "surface" diffusion.

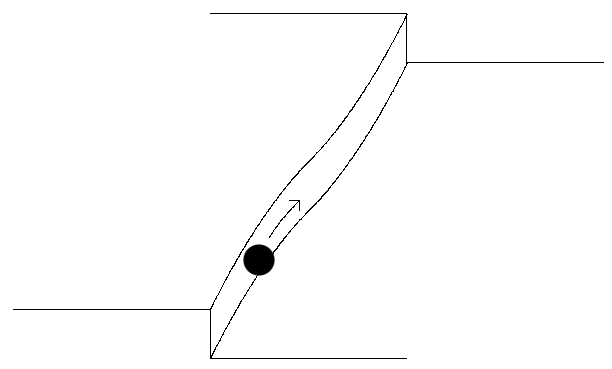

Figure 1: One-dimensional "surface" diffusion.

In developing our finite element method, we naturally divide our underlying problem into two parts: the adatom diffusion and the boundary evolution:

1. We derive a weak formulation for the time-dependent diffusion equation. In this formulation, the effect of the convection terms in the flux is implicitly included through the boundary conditions. To avoid the complexity in the spatial discretization near boundaries, in each time step, we extend the diffusion equation from terraces of same height to the whole computational domain. The extended equation is discretized using the linear finite element method. The resulting linear system is symmetric positive definite, and is solved by the conjugate gradient method. In order to trace the terrace height, all the elements are marked, and the marking is updated in each time step.

2. The geometric motion of the island boundaries includes both the mean curvature flow and the surface diffusion. It is treated in a variational formulation utilizing the curvature vector, and discretized by a semi-implicit front-tracking method using parametric finite elements. This method is adapted with modification from $[2,3,12]$.

We remark that the two-dimensional (2d) and the one-dimensional (1d) finite element meshes are essentially independent from each other. They are only coupled by corresponding right-hand side terms.

To obtain satisfactory computational results, meshes with sufficiently fine resolutions are needed for both the adatom diffusion equation and the boundary evolution equation. Thus, it is indispensable to use adaptivity in order for the method to be efficient. We use simple error indicators within an $h$-adaptive method to locally increase the spatial resolution. 
We apply our method to the following three test problems, and our numerical results demonstrate that the method is stable, efficient, and accurate enough to handle the island growth over a sufficiently long time period:

1. A pure geometrical problem of the evolution of the boundaries that is governed by either the motion by mean curvature or the motion by surface diffusion or by the combination of these two. Our numerical results show the expected smoothing properties of these motion laws;

2. A simplified model in which the coefficients of desorption and kinetic attachmentdetachment are set to be zero, so that islands cannot grow and the mass increases in time linearly due to a constant deposition flux rate. Our method is found to yield this mass balance consistently;

3. The stability of a growing circular island. This problem has been analyzed rigorously in [23]. Our method gives numerical result that agrees with the theory.

Besides these test problems, the method is used to study the influence of the onedimensional "surface" diffusion term in the velocity law on the growing of a single island. It is also applied to a situation with more than one island. At this stage, our method is not capable of handling topological changes of the moving boundaries in the nucleation and coalescence of adatom islands.

Recently, level-set based finite difference methods have been developed for the simulation of island dynamics in epitaxial growth $[8,10,16,26,32]$. Such a method is particularly efficient in handling topological changes. However, in treating the surface diffusion using such a method, fourth-order derivatives of a level-set function extended from the boundaries must be discretized by a finite difference scheme on a fixed Cartesian grid [11, 21, 24, 38]. An alternative method based on a phase-field approach was recently introduced in $[25,33]$. Due to the diffuse interface approximation, topological changes can be also handled by this approach. The use of an adequate degenerate mobility function in such a phase-field model furthermore allows the approximation of the Ehrlich-Schwoebel barrier. But the surface diffusion along edges is until now not included. While our method presented in this work cannot handle topological changes at the current stage, it has the advantage of exploiting the variational structure of the model to reduce the order of derivatives in discretization. This is evident, for instance, in the treatment of the surface diffusion term. Moreover, our method can be relatively easily extended to solve additional systems of equations such as the elasticity problem that can be important in determining the dynamics of heterogeneous epitaxial growth - the growth of material systems in which thin films and substrates have different lattice structures.

In Section 2, we describe the problem. In Section 3, we describe our methods of discretization for both the adatom diffusion equation and the boundary evolution equation. In Section 4, we describe implementational details such as the adaptivity, element marking, and numerical integration. In Section 5, we present our numerical results. Finally, in Section 6, we draw conclusions. 


\section{Problem description}

Consider the dynamics of adatom islands in an epitaxially growing thin film. An island or terrace is a portion of crystal layer that is one atomic layer higher than the immediate neighboring part of the film surface. Mathematically, we denote by $\Omega \subset \mathbb{R}^{2}$ the projected domain of the film surface in a two-dimensional Cartesian coordinate system, and assume that $\Omega$ is independent of time $t$. We denote also by $\Omega_{0}=\Omega_{0}(t) \subset \mathbb{R}^{2}$ the projected domain of the substrate or the exposed film surface with the smallest layer thickness, and by $\Omega_{i}=\Omega_{i}(t) \subset \mathbb{R}^{2}, i=1, \ldots, N$, that of the islands or terraces of height $i$ relative to $\Omega_{0}$ at time $t$, respectively. Thus, $N+1$ is the total number of layers that are exposed on the film surface. Note that, since the height of neighboring terraces differs only by one atomic layer, we conclude that

$$
\overline{\Omega_{i}(t)} \cap \overline{\Omega_{j}(t)}=\emptyset \text { if and only if }|i-j| \geq 2 .
$$

We denote further the corresponding island boundaries by

$$
\Gamma_{i}(t)=\overline{\Omega_{i}(t)} \cap \overline{\Omega_{i-1}(t)}, \quad i=1, \ldots, N,
$$

see Figure 2. We have that

$$
\bar{\Omega}=\bigcup_{i=0}^{N} \overline{\Omega_{i}(t)} .
$$

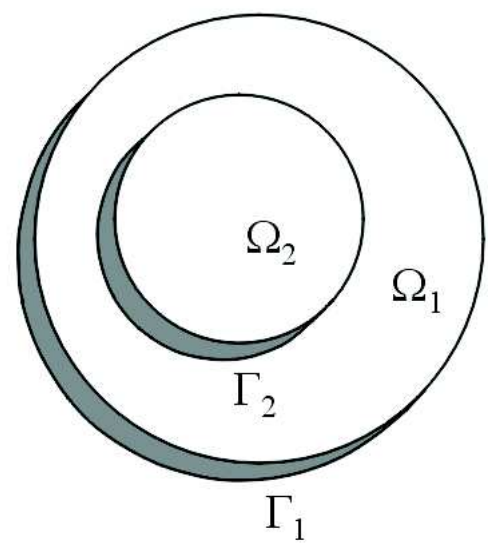

$\Omega_{0}$

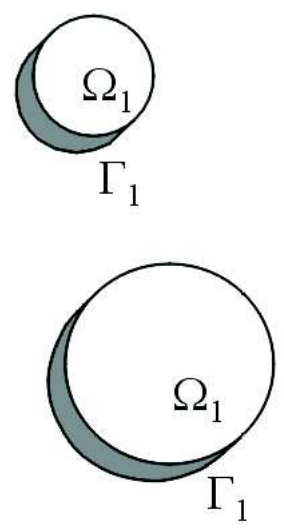

Figure 2: Schematic description of terraces $\Omega_{i}=\Omega_{i}(t)$ and boundaries $\Gamma_{i}=\Gamma_{i}(t)$.

Denote by $\rho_{i}=\rho_{i}(x, t)$ the adatom density on terrace $\Omega_{i}(t)(i=0, \ldots N)$ at time $t$. The adatom diffusion on a terrace is described by the diffusion equation for the adatom density $[6,9,15,22,28]$

$$
\partial_{t} \rho_{i}-D \Delta \rho_{i}=F-\tau^{-1} \rho_{i} \quad \text { in } \Omega_{i}(t), \quad i=0, \ldots, N
$$

where $D>0$ is the diffusion constant, $F$ is the deposition flux rate which shall be assumed to be a positive constant, and $\tau^{-1}>0$ is the constant desorption rate. 
We assume that the adatom density satisfies the following kinetic boundary conditions on the island boundary $\Gamma_{i}(t)$ for $i=1, \ldots, N[1,6,9,15,22,27,28]$ :

$$
\begin{aligned}
& q_{i}^{+}:=-D \nabla \rho_{i} \cdot \vec{n}_{i}-v_{i} \rho_{i}=k_{+}\left(\rho_{i}-\rho^{*}\left(1+\mu \kappa_{i}\right)\right), \\
& q_{i}^{-}:=D \nabla \rho_{i-1} \cdot \vec{n}_{i}+v_{i} \rho_{i-1}=k_{-}\left(\rho_{i-1}-\rho^{*}\left(1+\mu \kappa_{i}\right)\right),
\end{aligned}
$$

where: $q_{i}^{+}$and $q_{i}^{-}$are (normal) fluxes from the upper terrace $\Omega_{i}(t)$ and the lower terrace $\Omega_{i-1}(t)$, respectively, to the boundary $\Gamma_{i}(t) ; \vec{n}_{i}$ and $\kappa_{i}$ are the unit normal pointing from the upper to lower terrace and the curvature of the boundary $\Gamma_{i}(t)$, respectively; $v_{i}$ is the normal velocity of the island $\Gamma_{i}(t)$ with the convention that $v_{i}>0$ if the movement of $\Gamma_{i}(t)$ is in the direction of $\vec{n}_{i} ; k_{+}$and $k_{-}$are the kinetic attachment rates from the upper and lower terrace to the boundary $\Gamma_{i}(t)$, respectively; and $\rho^{*}$ and $\mu$ are two positive constants. In general, we have $0<k_{+} \leq k_{-}$by our notation, where the strict inequality $k_{+}<k_{-}$ models the Ehrlich-Scwhoebel effect. The constant $\rho^{*}$ can be either a thermodynamic equilibrium value or a kinetic steady state value, and the constant $\mu$ can be proportional to the stiffness of the boundary $\Gamma_{i}(t)$ or can come from a transition energy barrier, see $[1,6,7,9,27,28]$.

We note that the convection terms $\rho_{i} v_{i}$ and $\rho_{i-1} v_{i}$ in the fluxes $q_{i}^{+}$and $q_{i}^{-}$defined in (2.2) and (2.3), respectively, are often neglected in literature due to the smallness of the normal velocity. However, in some growth cases, these convection terms can be important to the growth stability $[14,19]$. In principle, they are necessary to obtain the conservation of mass in a region that includes a portion of the boundaries. Moreover, these terms can be incorporated naturally into a variational formulation of the diffusion problem, cf. Section 3.1.

For the motion of the moving boundaries, we assume the following law for the normal velocity $v_{i}$ of the island boundary $\Gamma_{i}(t)[9,18,22,27,28,30]$ :

$$
v_{i}=q_{i}^{+}+q_{i}^{-}+\nu \partial_{s s} \kappa_{i}
$$

where $\nu$ is a positive constant and $\partial_{s s}$ denotes the second-order tangential derivative along the boundaries. The term $\partial_{s s} \kappa_{i}$ represents the one-dimensional "surface" diffusion. The coefficient $\nu$ is related to the line tension and edge diffusion [9]. For $\nu=0$, the formula reduces to

$$
v_{i}=q_{i}^{+}+q_{i}^{-} .
$$

In this case, the diffusion along terrace boundaries is not taken into account.

We assume a flux-free boundary condition for the adatom density on the boundary of the film domain:

$$
\frac{\partial \rho_{0}}{\partial n}=0 \quad \text { at } \partial \Omega \text { for all } t>0
$$

where the normal derivative corresponds to the unit exterior normal $\vec{n}$ to the boundary $\partial \Omega$. We also assume that the initial islands $\Omega_{i}(0)(i=0, \ldots, N)$ along with their corresponding boundaries $\Gamma_{i}(0)(i=1, \ldots, N)$ are given. Moreover, we assume that initial adatom density is given by

$$
\rho_{i}(x, 0)=\bar{\rho}_{i}(x) \quad \forall x \in \Omega_{i}(0), \quad i=0, \ldots, N
$$

for some given function $\bar{\rho}_{i}$.

Finally, we assume no topological changes, i.e., islands neither nucleate nor coalesce, in the regime of island dynamics under consideration. 


\section{Variational formulation and finite element dis- cretization}

We derive a weak formulation for the time-dependent diffusion equation and use the first order implicit scheme to discretize the time derivative. In each time step:

1. We update the discrete boundaries by solving a geometric partial differential equation (PDE) based on the adatom densities and the discrete boundaries from the previous time step;

2. We solve the diffusion equation to update the adatom densities using the adatom densities and the computed discrete boundaries from the previous time step.

In Section 3.1, we describe the weak formulation for the time-dependent diffusion equation and the finite element discretization for the diffusion equation in each time step. In Section 3.2, we present our algorithm for the geometric PDE of the boundary evolution.

\subsection{Adatom diffusion}

Fix $i \in\{0,1, \ldots, N\}$. Assume that $\rho_{i}$ is smooth in $\Omega_{i}=\Omega_{i}(t)$. Multiplying both sides of the diffusion equation in (2.1) by a smooth, time-independent, test function $\phi$, and integrating by parts, we get

$$
\begin{gathered}
\int_{\Omega_{i}} \partial_{t} \rho_{i} \phi+\int_{\Omega_{i}} D \nabla \rho_{i} \cdot \nabla \phi-\int_{\Gamma_{i}} D \nabla \rho_{i} \cdot \vec{n}_{i} \phi+\int_{\Gamma_{i+1}} D \nabla \rho_{i} \cdot \vec{n}_{i+1} \phi \\
=\int_{\Omega_{i}} F \phi-\int_{\Omega_{i}} \tau^{-1} \rho_{i} \phi .
\end{gathered}
$$

Here and below, obvious modifications should be made for $i=0$ and $i=N$. Notice that for a moving smooth domain $\omega(t)$ and a smooth function $\xi(x, t)$ for $x \in \omega(t)$,

$$
\frac{d}{d t} \int_{\omega(t)} \xi=\int_{\omega(t)} \partial_{t} \xi+\int_{\partial \omega(t)} \xi u
$$

where $u$ is the normal velocity of the moving boundary $\partial \omega(t)$. Applying this formula to the corresponding term in (3.1) and using the boundary conditions (2.2) and (2.3), we obtain

$$
\begin{aligned}
& \frac{d}{d t} \int_{\Omega_{i}(t)} \rho_{i} \phi+\int_{\Omega_{i}(t)} D \nabla \rho_{i} \cdot \nabla \phi+\int_{\Omega_{i}(t)} \tau^{-1} \rho_{i} \phi+\int_{\Gamma_{i}(t)} k_{+}\left(\rho_{i}-\rho^{*}\left(1+\mu \kappa_{i}\right)\right) \phi \\
& \quad+\int_{\Gamma_{i+1}} k_{-}\left(\rho_{i}-\rho^{*}\left(1+\mu \kappa_{i+1}\right)\right) \phi=\int_{\Omega_{i}(t)} F \phi .
\end{aligned}
$$

Let $\triangle t>0$ be a small time step. Then,

$$
\frac{d}{d t} \int_{\Omega_{i}(t)} \rho_{i} \phi \approx \frac{1}{\triangle t}\left[\int_{\Omega_{i}(t)} \rho_{i}(x, t) \phi(x)-\int_{\Omega_{i}(t-\triangle t)} \rho_{i}(x, t-\triangle t) \phi(x)\right] .
$$

Now, split the time interval by discrete time instants $0=t_{0}<t_{1}<\ldots$ and define the time steps $\triangle t_{m}:=t_{m+1}-t_{m}(m=0,1, \ldots)$. Using the approximations $\Omega_{i}^{m} \approx \Omega_{i}\left(t_{m}\right)$ and $\Gamma_{i}^{m} \approx \Gamma_{i}\left(t_{m}\right)$, we have the following formulation of the time discretization problem. 
Problem 3.1 Set $\rho_{i}^{0}=\bar{\rho}_{i}(i=0, \ldots, N)$. For $m=0,1, \ldots$, find adatom densities $\rho_{i}^{m+1} \in H^{1}\left(\Omega_{i}^{m+1}\right)$ such that

$$
\begin{gathered}
\frac{1}{\triangle t_{m}}\left[\int_{\Omega_{i}^{m+1}} \rho_{i}^{m+1} \phi-\int_{\Omega_{i}^{m}} \rho_{i}^{m} \phi\right]+\int_{\Omega_{i}^{m+1}} D \nabla \rho_{i}^{m+1} \cdot \nabla \phi+\int_{\Omega_{i}^{m+1}} \tau^{-1} \rho_{i}^{m+1} \phi \\
+\int_{\Gamma_{i}^{m+1}} k_{+}\left(\rho_{i}^{m+1}-\rho^{*}\left(1+\mu \kappa_{i}^{m+1}\right)\right) \phi+\int_{\Gamma_{i+1}^{m+1}} k_{-}\left(\rho_{i}^{m+1}-\rho^{*}\left(1+\mu \kappa_{i+1}^{m+1}\right)\right) \phi \\
\quad=\int_{\Omega_{i}^{m+1}} F \phi \quad \forall \phi \in H^{1}\left(\Omega_{i}^{m+1}\right), \quad i=0, \ldots, N
\end{gathered}
$$

where $\kappa_{i}^{m+1}$ and $\kappa_{i+1}^{m+1}$ denote the curvature of $\Gamma_{i}^{m+1}$ and $\Gamma_{i+1}^{m+1}$, respectively.

At each time step, we need to solve an elliptic problem with curved boundaries. To avoid the complexity in the spatial discretization near such curved boundaries, we use an extension method. Let $\rho_{i}^{m}(m \geq 0)$ be the trivial extension of $\rho_{i}$ to the whole domain $\Omega$, i.e.,

$$
\rho_{i}^{m}(x)=\rho_{i}(x) \quad \text { for } x \in \Omega_{i}^{m} \quad \text { and } \quad \rho_{i}^{m}(x)=0 \quad \text { for } x \in \Omega \backslash \Omega_{i}^{m} .
$$

Furthermore, define for each $m \geq 0$ and $0 \leq i \leq N$

$$
D_{i, m}=\left\{\begin{array}{rl}
D & \text { in } \Omega_{i}^{m} \\
0 & \text { in } \Omega \backslash \Omega_{i}^{m}
\end{array} \quad, \quad F_{i, m}=\left\{\begin{array}{rl}
F & \text { in } \Omega_{i}^{m} \\
0 & \text { in } \Omega \backslash \Omega_{i}^{m}
\end{array}, \quad \tau_{i, m}^{-1}=\left\{\begin{array}{rl}
\tau^{-1} & \text { in } \Omega_{i}^{m} \\
0 & \text { in } \Omega \backslash \Omega_{i}^{m}
\end{array} .\right.\right.\right.
$$

Extend also the initial densities $\bar{\rho}_{i}$, still denoted by $\bar{\rho}_{i}$, by $\bar{\rho}_{i}=0$ in $\Omega \backslash \Omega_{i}(0)$. Now, replace $D, \tau^{-1}, F$, and $\Omega_{i}^{m}$ in Problem 3.1 by $D_{i, m+1}, \tau_{i, m+1}^{-1}, F_{i, m+1}$, and $\Omega$, and solve the corresponding problem on the whole domain for all test function $\phi$ defined on $\Omega$ :

$$
\begin{gathered}
\int_{\Omega} \frac{\rho_{i}^{m+1}-\rho_{i}^{m}}{\Delta t_{m}} \phi+\int_{\Omega} D_{i, m+1} \nabla \rho_{i}^{m+1} \cdot \nabla \phi+\int_{\Omega} \tau_{i, m+1}^{-1} \rho_{i}^{m+1} \phi \\
+\int_{\Gamma_{i}^{m+1}} k_{+}\left(\rho_{i}^{m+1}-\rho^{*}\left(1+\mu \kappa_{i}\right)\right) \phi+\int_{\Gamma_{i+1}^{m+1}} k_{-}\left(\rho_{i}^{m+1}-\rho^{*}\left(1+\mu \kappa_{i+1}\right)\right) \phi \\
=\int_{\Omega} F_{i, m+1} \phi \quad \forall \phi \in H^{1}(\Omega), \quad i=0, \ldots, N .
\end{gathered}
$$

To discretize in space, let $\mathcal{T}_{h}^{m}$ be an admissible shape-regular triangulation of $\Omega$ at time instant $t_{m}$ [5, II.5]. Notice that we do not assume that the triangulation is uniform, allowing thus for highly graded local mesh refinement. We will use the finite element space of globally continuous, piecewise linear elements

$$
\mathbb{V}_{h}^{m}=\left\{v_{h} \in C^{0}(\bar{\Omega}):\left.v_{h}\right|_{T} \in \mathbb{P}_{1} \quad \forall T \in \mathcal{T}_{h}^{m}\right\},
$$

where $\mathbb{P}_{1}$ denotes the set of all polynomials of total degree $\leq 1$.

Denote by $P_{m}: C^{0}(\bar{\Omega}) \rightarrow \mathbb{V}_{h}^{m}$ the usual Lagrange interpolation operator. With this setting, the space discretization of Problem 3.1 based on our method of extension can be summarized as follows: 
Problem 3.2 Let $\rho_{i, h}^{0}=P_{0} \bar{\rho}_{i}(i=0, \ldots, N)$. For $m=0,1, \ldots$, determine the discrete adatom densities $\rho_{i, h}^{m+1} \in \mathbb{V}_{h}^{m+1}$ for $i=0, \ldots, N$ by

$$
\begin{aligned}
& \int_{\Omega} \frac{\rho_{i, h}^{m+1}-\rho_{i, h}^{m}}{\triangle t_{m}} \phi_{h}+\int_{\Omega} D_{i, m+1} \nabla \rho_{i, h}^{m+1} \cdot \nabla \phi_{h}+\int_{\Gamma_{i, h}^{m+1}} k_{+}\left(\rho_{i, h}^{m+1}-\rho^{*}\left(1+\mu \kappa_{i, h}^{m+1}\right)\right) \phi_{h} \\
& +\int_{\Gamma_{i+1, h}^{m+1}} k_{-}\left(\rho_{i, h}^{m+1}-\rho^{*}\left(1+\mu \kappa_{i+1, h}^{m+1}\right)\right) \phi_{h}+\int_{\Omega} \tau_{i, m+1}^{-1} \rho_{i, h}^{m+1} \phi_{h}=\int_{\Omega} F_{i, m+1} \phi_{h} \quad \forall \phi_{h} \in \mathbb{V}_{h}^{m+1}
\end{aligned}
$$

with $\kappa_{i, h}^{m+1}$ and $\kappa_{i+1, h}^{m+1}$ the discrete curvatures of $\Gamma_{i, h}^{m+1}$ and $\Gamma_{i+1, h}^{m+1}$, respectively, defined in Problem 3.5 below.

In the rest of this subsection, we fix a time step $m$ and drop the subscript and superscript $m+1$, when no confusion arises. Let $\left(\phi_{k}\right)_{k=1}^{L}$ be the standard nodal basis of the finite element space $\mathbb{V}_{h}$, where $L$ is the dimension of $\mathbb{V}_{h}$. Expand $\rho_{i, h}^{m+1}$ as

$$
\rho_{i, h}^{m+1}=\sum_{k=1}^{L} r_{k} \phi_{k}
$$

for some $R_{i}=\left(r_{1}, \ldots, r_{L}\right)^{t} \in \mathbb{R}^{L}$, where the superscript $t$ denotes the transpose matrix. Define the following mass and stiffness matrices and load vectors:

$$
\begin{array}{llll}
\boldsymbol{M}=\left(M_{k l}\right), & M_{k l}=\left(\phi_{k}, \phi_{l}\right) ; & \boldsymbol{M}_{i}=\left(M_{i, k l}\right), & M_{i, k l}=\left(\tau_{i}^{-1} \phi_{k}, \phi_{l}\right) ; \\
\boldsymbol{M}^{\Gamma_{i}}=\left(M_{k l}^{\Gamma_{i}}\right), & M_{k l}^{\Gamma_{i}}=\left\langle\phi_{k}, \phi_{l}\right\rangle_{\Gamma_{i}} ; & \boldsymbol{A}_{i}=\left(A_{i, k l}\right), & A_{i, k l}=\left(D_{i} \nabla \phi_{k}, \nabla \phi_{l}\right) ; \\
\boldsymbol{F}_{i}=\left(F_{i, l}\right), & F_{i, l}=\left(F_{i}, \phi_{l}\right) ; & \boldsymbol{F}^{\Gamma_{i}}=\left(F_{l}^{\Gamma_{i}}\right), & F_{l}^{\Gamma_{i}}=\left\langle\rho^{*}\left(1+\mu \kappa_{i, h}\right), \phi_{l}\right\rangle_{\Gamma_{i}} ;
\end{array}
$$

where the index ranges are $1 \leq k, l \leq L$ and $\langle\cdot, \cdot\rangle_{\Gamma_{i}}$ stands for the $L^{2}$ inner product over the current interface $\Gamma_{i}=\Gamma_{i, h}$ and $(\cdot, \cdot)$ stands for the $L^{2}$ inner product over the domain $\Omega$. The following algorithm is the matrix form of Problem 3.2.

Algorithm 3.1 For $m=0,1, \ldots$, find $R_{i}^{m+1} \in \mathbb{R}^{L}$ such that

$$
\begin{aligned}
\frac{1}{\triangle t_{m}} \boldsymbol{M} R_{i}^{m+1} & +\boldsymbol{A}_{i} R_{i}^{m+1}+\boldsymbol{M}_{i} R_{i}^{m+1}+k_{+} \boldsymbol{M}^{\Gamma_{i}} R_{i}^{m+1}+k_{-} \boldsymbol{M}^{\Gamma_{i+1}} R_{i}^{m+1} \\
& =\boldsymbol{F}_{i}+k_{+} \boldsymbol{F}^{\Gamma_{i}}+k_{-} \boldsymbol{F}^{\Gamma_{i+1}}+\frac{1}{\triangle t_{m}} \boldsymbol{M} R_{i}^{m}, \quad i=0, \ldots, N .
\end{aligned}
$$

We introduce the following quantities defined on the nodes on the boundaries $\Gamma_{i, h}$ :

$$
\gamma_{i}:=k_{+}\left(\rho_{i}-\rho^{*}\right)+k_{-}\left(\rho_{i-1}-\rho^{*}\right)=k_{+}\left(\left.\rho_{i, h}\right|_{\Gamma_{i, h}}-\rho^{*}\right)+k_{-}\left(\left.\rho_{i-1, h}\right|_{\Gamma_{i, h}}-\rho^{*}\right) .
$$

These quantities will enter in the subproblem of moving boundaries.

Remark 3.1 (Number of subproblems) In actual computations, the number of diffusion equations to be solved can be reduced from the number of layers $N+1$ to at most 2. Indeed, since odd-labeled (or even-labeled) terraces are non-contiguous, it is enough to work with a single function $\rho_{\text {odd }}$ for all odd $i$ 's and a single function $\rho_{\text {even }}$ for all even $i$ 's. 


\subsection{Boundary evolution}

Adding the two boundary conditions $(2.2)$ and $(2.3)$ at $\Gamma_{i}(t)$, we get

$$
q_{i}^{+}+q_{i}^{-}=k_{+}\left(\rho_{i}-\rho^{*}\left(1+\mu \kappa_{i}\right)\right)+k_{-}\left(\rho_{i-1}-\rho^{*}\left(1+\mu \kappa_{i}\right)\right) .
$$

This, together with the velocity formula (2.4), leads to the geometric PDE

$$
v_{i}=k_{+}\left(\rho_{i}-\rho^{*}\right)+k_{-}\left(\rho_{i-1}-\rho^{*}\right)-\left(k_{+}+k_{-}\right) \rho^{*} \mu \kappa_{i}+\nu \partial_{s s} \kappa_{i} .
$$

This equation can be interpreted as an equation for surface diffusion with lower order terms if $\nu>0$, or for the mean curvature flow with a forcing term if $\nu=0$. For solving such a highly nonlinear 4 th order $(\nu>0)$ or 2 nd order $(\nu=0)$ equation, we adapt with modification a variational formulation introduced for surface diffusion by Bänsch et al [3], cf. also [12]. By introducing the position vector $\vec{x}_{i}$, the curvature vector $\vec{\kappa}_{i}$, and the velocity vector $\vec{v}_{i}$, a system of equations for $\vec{\kappa}_{i}, \kappa_{i}, v_{i}$, and $\vec{v}_{i}$ can be derived from (3.5). By the geometric expression $\vec{\kappa}_{i}=-\partial_{s s} \vec{x}_{i}$, the velocity law (3.5), and the relations between the vector-valued and scalar quantities $\kappa_{i}=\vec{\kappa}_{i} \cdot \vec{n}_{i}$ and $\vec{v}_{i}=v_{i} \vec{n}_{i}$, we obtain

$$
\begin{aligned}
\vec{\kappa}_{i} & =-\partial_{s s} \vec{x}_{i}, \\
\kappa_{i} & =\vec{\kappa}_{i} \cdot \vec{n}_{i}, \\
v_{i} & =\gamma_{i}-\beta \kappa_{i}+\alpha \partial_{s s} \kappa_{i}, \\
\vec{v}_{i} & =v_{i} \vec{n}_{i} .
\end{aligned}
$$

Here, in addition to $\gamma_{i}$ introduced in (3.3), we use the coefficients

$$
\alpha=\nu \geq 0 \quad \text { and } \quad \beta=\left(k_{+}+k_{-}\right) \mu \rho^{*} \geq 0 .
$$

Consider the discrete time instant $t_{m}$ and time step $\Delta t_{m}:=t_{m+1}-t_{m}$ as in Section 3.1. We represent the next boundary $\Gamma_{i}^{m+1}$ in terms of the current boundary $\Gamma_{i}^{m}$ by updating the position vectors

$$
\vec{x}_{i} \leftarrow \vec{x}_{i}+\triangle t_{m} \vec{v}_{i}
$$

In the time discretization, all the geometric quantities such as $\vec{n}_{i}$ and $\kappa_{i}$, and the differentiation $\partial_{s s}$ are evaluated on the current boundary $\Gamma_{i}^{m}$. In contrast to the geometric quantities, the unknowns $\vec{\kappa}_{i}, \kappa_{i}, v_{i}$, and $\vec{v}_{i}$ are treated implicitly. In particular, in view of (3.10), we define

$$
\vec{\kappa}_{i}^{m+1}:=-\partial_{s s}\left(\vec{x}_{i}^{m}+\triangle t_{m} \vec{v}_{i}^{m+1}\right) .
$$

To derive a weak formulation, we proceed similarly as in [12]: multiply (3.7), (3.8), (3.9), and (3.11) by test functions $\vec{\psi} \in \vec{H}^{1}\left(\Gamma_{i}\right)$ and $\psi \in H^{1}\left(\Gamma_{i}\right)$, and use integration by parts for the second order operator $\partial_{s s}$. For simplicity, we have hereafter dropped the superscript $m+1$ for the unknowns $\vec{\kappa}_{i}^{m+1}$, etc. Furthermore, using the notation $\langle\cdot, \cdot\rangle$ for the $L^{2}$ inner product over the current interfaces $\Gamma_{i}^{m}$, we arrive at the following set of semi-implicit equations: 
Problem 3.3 For $m=1,2, \ldots$ find $\vec{\kappa}_{i} \in \vec{H}^{1}\left(\Gamma_{i}^{m}\right), \kappa_{i} \in H^{1}\left(\Gamma_{i}^{m}\right), v_{i} \in H^{1}\left(\Gamma_{i}^{m}\right)$, and $\vec{v}_{i} \in \vec{H}^{1}\left(\Gamma_{i}^{m}\right)$ such that

$$
\begin{aligned}
\left\langle\vec{\kappa}_{i}, \vec{\psi}\right\rangle-\triangle t_{m}\left\langle\partial_{s} \vec{v}_{i}, \partial_{s} \vec{\psi}\right\rangle & =\left\langle\partial_{s} \vec{x}_{i}^{m}, \partial_{s} \vec{\psi}\right\rangle & & \forall \vec{\psi} \in \vec{H}^{1}\left(\Gamma_{i}^{m}\right), \\
\left\langle\kappa_{i}, \psi\right\rangle-\left\langle\vec{\kappa}_{i} \cdot \vec{n}_{i}, \psi\right\rangle & =0 & & \forall \psi \in H^{1}\left(\Gamma_{i}^{m}\right), \\
\left\langle v_{i}, \psi\right\rangle+\alpha\left\langle\partial_{s} \kappa_{i}, \partial_{s} \psi\right\rangle+\beta\left\langle\kappa_{i}, \psi\right\rangle & =\left\langle\gamma_{i}, \psi\right\rangle & & \forall \psi \in H^{1}\left(\Gamma_{i}^{m}\right), \\
\left\langle\vec{v}_{i}, \vec{\psi}\right\rangle-\left\langle v_{i} \vec{n}_{i}, \vec{\psi}\right\rangle & =0 & & \forall \vec{\psi} \in \vec{H}^{1}\left(\Gamma_{i}^{m}\right) .
\end{aligned}
$$

Note that in the above formulation, the adatom densities on the upper and lower terraces $\rho_{i}$ and $\rho_{i-1}$, respectively, are needed only for computing $\gamma_{i}$ which is defined in $(3.3)$.

To discretize in space, we consider a polygonal curve $\Gamma_{i, h}^{m}$ approximating $\Gamma_{i}$ at time $t_{m}$. The polygonal segments are thought of as finite elements. Making a customary abuse of terminology, we identify these segments with the corresponding finite element partition. We denote by $\vec{n}_{i, h}$ the unit normal to $\Gamma_{i, h}^{m}$ pointing to the lower terrace. It is discontinuous across inter-element boundaries. Denote by $\mathbb{W}_{h}^{m} \subseteq H^{1}\left(\Gamma_{i, h}^{m}\right)$ the finite element space of globally continuous, piecewise linear functions with corresponding nodal basis functions $\left(\psi_{k}\right)_{k=1}^{K}$, where $K$ is the number of degrees of freedom. By $\overrightarrow{\mathbb{W}}_{h}^{m} \subseteq \vec{H}^{1}\left(\Gamma_{i, h}^{m}\right)$ we denote the finite element space of vector-valued functions with nodal basis functions $\left(\vec{\psi}_{k}^{q}\right)_{k=1, \ldots, K}^{q=1,2}$, where $\vec{\psi}_{k}^{q}=\psi_{k} \vec{e}_{q}$ with $\psi_{k}$ the scalar basis function defined above and $\left(\vec{e}_{1}, \vec{e}_{2}\right)$ the standard basis in $\mathbb{R}^{2}$.

Upon expanding the functions $\vec{\kappa}_{i}, \kappa_{i}, v_{i}, \vec{v}_{i}$ in terms of the basis functions and testing against all discrete test functions, a discretization of Problem 3.3 is now at hand.

Problem 3.4 Find $\vec{\kappa}_{i, h}=\vec{\kappa}_{i, h}^{m} \in \overrightarrow{\mathbb{W}}_{h}^{m}, \kappa_{i, h}=\kappa_{i, h}^{m} \in \mathbb{W}_{h}^{m}, v_{i, h}=v_{i, h}^{m} \in \mathbb{W}_{h}^{m}$, and $\vec{v}_{i, h}=$ $\vec{v}_{i, h}^{m} \in \overrightarrow{\mathbb{W}}_{h}^{m}$ such that

$$
\begin{aligned}
\left\langle\vec{\kappa}_{i, h}, \vec{\psi}_{h}\right\rangle-\triangle t_{m}\left\langle\partial_{s} \vec{v}_{i, h}, \partial_{s} \vec{\psi}_{h}\right\rangle & =\left\langle\partial_{s} \vec{x}_{i}^{m}, \partial_{s} \vec{\psi}_{h}\right\rangle & & \forall \vec{\psi}_{h} \in \overrightarrow{\mathbb{W}}_{h}^{m}, \\
\left\langle\kappa_{i, h}, \psi_{h}\right\rangle-\left\langle\vec{\kappa}_{i, h} \cdot \vec{n}_{i, h}, \psi_{h}\right\rangle & =0 & & \forall \psi_{h} \in \mathbb{W}_{h}^{m}, \\
\left\langle v_{i, h}, \psi_{h}\right\rangle+\alpha\left\langle\partial_{s} \kappa_{i, h}, \partial_{s} \psi_{h}\right\rangle+\beta\left\langle\kappa_{i, h}, \psi_{h}\right\rangle & =\left\langle\gamma_{i}, \psi_{h}\right\rangle & & \forall \psi_{h} \in \mathbb{W}_{h}^{m}, \\
\left\langle\vec{v}_{i, h}, \vec{\psi}_{h}\right\rangle-\left\langle v_{i, h} \vec{n}_{i, h}, \vec{\psi}_{h}\right\rangle & =0 & & \forall \vec{\psi}_{h} \in \overrightarrow{\mathbb{W}}_{h}^{m} .
\end{aligned}
$$

This discrete scheme is now translated into a matrix-vector system by using the nodal bases $\left(\psi_{k}\right)$ and $\left(\vec{\psi}_{k}^{q}\right)$ to obtain the mass, stiffness, and normal matrices, and the load vector

$$
\begin{array}{llll}
\boldsymbol{M}=\left(M_{k l}\right), & M_{k l}=\left\langle\psi_{k}, \psi_{l}\right\rangle ; & \overrightarrow{\boldsymbol{M}}=\left(\vec{M}_{k l}\right), & \vec{M}_{k l}=\left(M_{k l}^{q r}\right)=\left(\delta_{q r} M_{k l}\right) ; \\
\boldsymbol{A}=\left(A_{k l}\right), & A_{k l}=\left\langle\partial_{s} \psi_{k}, \partial_{s} \psi_{l}\right\rangle ; & \overrightarrow{\boldsymbol{A}}=\left(\vec{A}_{k l}\right), & \vec{A}_{k l}=\left(A_{k l}^{q r}\right)=\left(\delta_{q r} A_{k l}\right) ; \\
G=\left(G_{k}\right), & G_{k}=\left\langle\gamma_{i}, \psi_{k}\right\rangle ; & \overrightarrow{\boldsymbol{N}}=\left(\vec{N}_{k l}\right), & \vec{N}_{k l}=\left(N_{k l}^{q}\right)=\left(\left\langle\psi_{k}, \psi_{l} n_{i, h}^{q}\right\rangle\right) ;
\end{array}
$$

where the index ranges are $1 \leq k, l \leq K$ and $1 \leq q, r \leq 2, \delta_{q r}=\vec{e}_{q} \cdot \vec{e}_{r}$ is the Kronecker symbol, and $n_{i, h}^{q}=\vec{n}_{i, h} \cdot \vec{e}_{q}$ is the $q$-th spatial component of the normal.

An alternative way of looking at the system is given by ordering the coefficient vector $\left(x_{k}^{q}\right)_{k=1, \ldots, K}^{q=1,2}$ corresponding to an element $\vec{x}_{h} \in \overrightarrow{\mathbb{W}}_{h}^{m}$ as a column vector $\vec{X}=\left(X_{1}^{t}, X_{2}^{t}\right)^{t}$, 
where $X_{q}$ are the (column) vectors of coefficients corresponding to spatial components of $\vec{X}$. With this description, we can write

$$
\vec{A}=\left(\begin{array}{cc}
A & 0 \\
0 & A
\end{array}\right), \quad \vec{M}=\left(\begin{array}{cc}
M & 0 \\
0 & M
\end{array}\right), \quad \vec{N}=\left(\begin{array}{l}
N_{1} \\
N_{2}
\end{array}\right)
$$

where all the entries are square matrices in $\mathbb{R}^{K \times K}$, with the spatial components $\boldsymbol{N}_{q}=$ $\left(N_{k l}^{q}\right)$ of the normal matrix being some kind of "weighted" mass matrices. The linear system takes now the following matrix form.

Algorithm 3.2 Find $\vec{K}_{i}, \vec{V}_{i} \in \mathbb{R}^{2 \times K}, K_{i}, V_{i} \in \mathbb{R}^{K}$ such that

$$
\left(\begin{array}{cccc}
\overrightarrow{\boldsymbol{M}} & \mathbf{0} & \mathbf{0} & -\overrightarrow{\boldsymbol{N}} \\
\mathbf{0} & \boldsymbol{M} & -\overrightarrow{\boldsymbol{N}}^{t} & \mathbf{0} \\
-\triangle t_{m} \overrightarrow{\boldsymbol{A}} & \mathbf{0} & \overrightarrow{\boldsymbol{M}} & \mathbf{0} \\
\mathbf{0} & \alpha \boldsymbol{A}+\beta \boldsymbol{M} & \mathbf{0} & \boldsymbol{M}
\end{array}\right)\left(\begin{array}{c}
\vec{V}_{i} \\
K_{i} \\
\vec{K}_{i} \\
V_{i}
\end{array}\right)=\left(\begin{array}{c}
0 \\
0 \\
\overrightarrow{\boldsymbol{A}} \vec{X}_{i}^{m} \\
G
\end{array}\right)
$$

With this arrangement, a Schur complement equation for $\vec{K}_{i}, V_{i}$ reads

$$
\boldsymbol{S}\left(\begin{array}{c}
\vec{K}_{i} \\
V_{i}
\end{array}\right)=\left(\begin{array}{c}
\overrightarrow{\boldsymbol{A}} \vec{X}_{i}^{m} \\
G
\end{array}\right)
$$

where

$$
\begin{aligned}
\boldsymbol{S} & =\left(\begin{array}{cc}
\overrightarrow{\boldsymbol{M}} & \mathbf{0} \\
\mathbf{0} & \boldsymbol{M}
\end{array}\right)-\left(\begin{array}{cc}
-\triangle t_{m} \overrightarrow{\boldsymbol{A}} & \mathbf{0} \\
\mathbf{0} & \alpha \boldsymbol{A}+\beta \boldsymbol{M}
\end{array}\right)\left(\begin{array}{cc}
\vec{M} & \mathbf{0} \\
\mathbf{0} & \boldsymbol{M}
\end{array}\right)^{-1}\left(\begin{array}{cc}
\mathbf{0} & -\overrightarrow{\boldsymbol{N}} \\
-\overrightarrow{\boldsymbol{N}}^{t} & \mathbf{0}
\end{array}\right) \\
& =\left(\begin{array}{cc}
\overrightarrow{\boldsymbol{M}} & -\triangle t_{m} \overrightarrow{\boldsymbol{A}} \overrightarrow{\boldsymbol{M}}^{-1} \overrightarrow{\boldsymbol{N}} \\
\alpha \boldsymbol{A} \boldsymbol{M}^{-1} \overrightarrow{\boldsymbol{N}}^{t}+\beta \overrightarrow{\boldsymbol{N}}^{t} & \boldsymbol{M}
\end{array}\right) .
\end{aligned}
$$

The above formulation in turn gives rise to the final Schur complement equation for the single unknown $V_{i}$ :

$$
\begin{aligned}
\left(\triangle t_{m}\left(\alpha \boldsymbol{A} \boldsymbol{M}^{-1} \overrightarrow{\boldsymbol{N}}^{t}+\beta \overrightarrow{\boldsymbol{N}}^{t}\right) \overrightarrow{\boldsymbol{M}}^{-1} \overrightarrow{\boldsymbol{A}} \overrightarrow{\boldsymbol{M}}^{-1} \overrightarrow{\boldsymbol{N}}+\boldsymbol{M}\right) V_{i} & \\
& =G-\left(\alpha \boldsymbol{A} \boldsymbol{M}^{-1} \overrightarrow{\boldsymbol{N}}^{t}+\beta \overrightarrow{\boldsymbol{N}}^{t}\right) \overrightarrow{\boldsymbol{M}}^{-1} \overrightarrow{\boldsymbol{A}} \vec{X}_{i}^{m}
\end{aligned}
$$

In the case of $\nu=0$ (i.e., $\alpha=0$ ), the equation for $V_{i}$ reduces to

$$
\left(\triangle t_{m} \beta \overrightarrow{\boldsymbol{N}}^{t} \overrightarrow{\boldsymbol{M}}^{-1} \overrightarrow{\boldsymbol{A}} \overrightarrow{\boldsymbol{M}}^{-1} \overrightarrow{\boldsymbol{N}}+\boldsymbol{M}\right) V_{i}=G-\beta \overrightarrow{\boldsymbol{N}}^{t} \overrightarrow{\boldsymbol{M}}^{-1} \overrightarrow{\boldsymbol{A}} \vec{X}_{i}^{m}
$$

The linear systems in both cases, (3.13) and (3.14), are uniquely solvable. We show this for (3.13), the result for (3.14) follows as a special case. Introducing the symmetric non-negative matrix

$$
\boldsymbol{L}=\overrightarrow{\boldsymbol{N}}^{t} \overrightarrow{\boldsymbol{M}}^{-1} \overrightarrow{\boldsymbol{A}} \overrightarrow{\boldsymbol{M}}^{-1} \overrightarrow{\boldsymbol{N}}=\sum_{q=1}^{2} \boldsymbol{N}_{q} \boldsymbol{M}^{-1} \boldsymbol{A} \boldsymbol{M}^{-1} \boldsymbol{N}_{q}
$$


The matrix in the left hand side of (3.13), which we denote by $\boldsymbol{T}$, can be written as

$$
\boldsymbol{T}=\triangle t_{m}\left(\alpha \boldsymbol{A} \boldsymbol{M}^{-1}+\beta \boldsymbol{I}\right) \boldsymbol{L}+\boldsymbol{M} .
$$

It is enough to show that $\boldsymbol{T}$ is invertible. To this end, we show that if for some $V \in \mathbb{R}^{K}$ we have $\boldsymbol{T} V=0$ then $V$ must be 0 . Assuming $\boldsymbol{T} V=0$ it ensues that $W^{t} \boldsymbol{T} V=0$, for any $W \in \mathbb{R}^{K}$. In particular, for $W=\boldsymbol{M}^{-1} \boldsymbol{L} V$ we obtain

$$
0=\triangle t_{m} \alpha V^{t} \boldsymbol{L} \boldsymbol{M}^{-1} \boldsymbol{A} \boldsymbol{M}^{-1} \boldsymbol{L} V+\triangle t_{m} \beta V^{t} \boldsymbol{L} \boldsymbol{M}^{-1} \boldsymbol{L} V+V^{t} \boldsymbol{L} V \geq 0,
$$

by symmetry and non-negativity of the involved matrices. It follows that $V^{t} \boldsymbol{L} V=0$. Again by the symmetry and non-negativity of $\boldsymbol{L}$ we conclude that $\boldsymbol{L} V=0$. This implies that $\boldsymbol{M V}=\boldsymbol{T} V$ which we assumed to be zero. Since $\boldsymbol{M}$ is invertible, it follows that $V=0$. Therefore $\boldsymbol{T}$ is invertible. Backsubstituting $V_{i}$ into the linear system in Algorithm 3.2 and then solving for $\vec{K}_{i}$ and likewise for $K_{i}$ and $\vec{V}_{i}$ we see that the linear system is uniquely solvable.

Once $V_{i}$ is obtained by solving (3.13) or (3.14), the unknown $\vec{V}_{i}$ is easily computed by solving

$$
\vec{M} \vec{V}_{i}=\vec{N} V_{i},
$$

$\overrightarrow{\boldsymbol{M}}$ being invertible, and then $\vec{X}_{i}$ is updated through

$$
\vec{X}_{i} \leftarrow \vec{X}_{i}+\triangle t_{m} \vec{V}_{i}
$$

The curvature, which is needed as data in the adatom diffusion problem, is now computed for accuracy reasons on the new interface $\Gamma_{i, h}^{m+1}$ instead of the old interface $\Gamma_{i, h}^{m}$. To this end, we use the same formulation as above but with all the geometric quantities defined for $\Gamma_{i, h}^{m+1}$ replacing $\Gamma_{i, h}^{m}$. We obtain the following problem formulation:

Problem 3.5 Find $\vec{\kappa}_{i, h}=\vec{\kappa}_{i, h}^{m+1} \in \overrightarrow{\mathbb{W}}_{h}^{m+1}$ and $\kappa_{i, h}=\kappa_{i, h}^{m+1} \in \mathbb{W}_{h}^{m+1}$ with

$$
\begin{aligned}
\left\langle\vec{\kappa}_{i, h}, \vec{\psi}_{h}\right\rangle & =\left\langle\partial_{s} \vec{x}_{i}^{m+1}, \partial_{s} \vec{\psi}_{h}\right\rangle & & \forall \vec{\psi}_{h} \in \overrightarrow{\mathbb{W}}_{h}^{m+1}, \\
\left\langle\kappa_{i, h}, \psi_{h}\right\rangle-\left\langle\vec{\kappa}_{i, h} \cdot \vec{n}_{i, h}, \psi_{h}\right\rangle & =0 & & \forall \psi_{h} \in \mathbb{W}_{h}^{m+1} .
\end{aligned}
$$

Again, the system can be written equivalently in matrix form, where the matrices are now defined in terms of the basis functions on $\Gamma_{i, h}^{m+1}$ :

Algorithm 3.3 Find $\vec{K}_{i}$ and $K_{i}$ such that

$$
\left(\begin{array}{cc}
\boldsymbol{M} & -\overrightarrow{\boldsymbol{N}}^{t} \\
0 & \overrightarrow{\boldsymbol{M}}
\end{array}\right)\left(\begin{array}{c}
K_{i} \\
\vec{K}_{i}
\end{array}\right)=\left(\begin{array}{c}
0 \\
\overrightarrow{\boldsymbol{A}} \vec{X}_{i}^{m+1}
\end{array}\right) .
$$

This leads to

$$
K_{i}=-\boldsymbol{M}^{-1} \overrightarrow{\boldsymbol{N}} \overrightarrow{\boldsymbol{M}}^{-1} \overrightarrow{\boldsymbol{A}} \vec{X}_{i}^{m+1} .
$$

In summary, the subproblem of boundary evolution consists of solving $N$ decoupled problems for each interface $\Gamma_{i, h}, i=1, \ldots, N$, according to Algorithms 3.2 and 3.3. For the adatom diffusion problem the new interfaces $\Gamma_{i, h}^{m+1}$ and the curvatures $\kappa_{i, h}^{m+1}$ will enter. 


\section{Implementation}

We implement our numerical method using ALBERT, an adaptive finite element software for scientific computation [35]. The program for the two dimensional adatom diffusion and that for the one dimensional boundary evolution are coupled via a TCP/IP port. All matrices are assembled using the standard assembling tools of ALBERT as well as the methods described below.

\subsection{Adaptivity for adatom diffusion}

To obtain satisfactory computational results, a mesh with a sufficiently fine resolution near the island boundaries is needed. Noting that a uniform refinement would be prohibitive from the computational point of view, we are naturally led to adopt local mesh refinement. Since the island boundaries are moving, it is indispensable to use some adaptive strategy for local mesh refinement and coarsening. At every time step, the $2 \mathrm{~d}$ finite element mesh from the previous time step is locally refined and/or coarsened. Every element in the mesh is marked for being refined, coarsened, or left unchanged. The actual mesh modification is then performed within the programming environment ALBERT [35] that uses the so called bisection method to locally modify meshes.

The criterion for refinement is purely geometric: the $2 \mathrm{~d}$ mesh is refined near the boundaries $\Gamma_{i, h}^{m}$ until the mesh size for both the $1 \mathrm{~d}$ and $2 \mathrm{~d}$ meshes are locally of the same order. More precisely, the $2 \mathrm{~d}$ mesh is refined until no $1 \mathrm{~d}$ element is fully contained in any $2 \mathrm{~d}$ element. This criterion can be easily satisfied by traversing $\Gamma_{i, h}^{m}$ and refining all visited elements of the $2 \mathrm{~d}$ mesh $\mathcal{T}_{\Omega}^{m}$ with the element size larger than the $1 \mathrm{~d}$ mesh size. Elements may be further refined to satisfy the assumptions (A) and (B) in Section 4.3.

We define $\rho_{h}(x, t)$ as the overall adatom density by

$$
\rho_{h}(x, t)=\rho_{i, h}(x, t) \quad \text { for } x \in \Omega_{i}(t), \quad i=0, \ldots, N .
$$

Here and below in this section, when no confusion arises, we use $\Omega_{i}=\Omega_{i}(t)$ to denote both the original domain and its finite element approximation determined by $\Gamma_{i, h}(t)$. We use an $L^{2}$-like error indicator for local mesh coarsening. For every element $T$, we define

$$
\eta_{T}\left(\rho_{h}\right):=\left(\sum_{e \in \partial T} \int_{e} h^{3}\left|\left[\frac{\partial \rho_{h}}{\partial n_{e}}\right]\right|^{2}\right)^{1 / 2},
$$

where $\left[\frac{\partial \rho_{h}}{\partial n_{e}}\right]$ denotes the jump of the normal derivative of $\rho_{h}$ across an edge $e \subset \partial T$. This can be used to define an indicator for the error $\left\|\rho-\rho_{h}\right\|$ on the whole domain

$$
\eta\left(\rho_{h}\right):=\left(\sum_{T \in \mathcal{T}_{\Omega}^{m}} \eta_{T}^{2}\left(\rho_{h}\right)\right)^{1 / 2} .
$$

The criterion for coarsening is based on an equidistribution strategy, which attempts to enforce $\eta_{T}\left(\rho_{h}\right)=\eta_{T^{\prime}}\left(\rho_{h}\right)$ for all $T, T^{\prime} \in \mathcal{T}_{\Omega}^{m}$. If this condition were enforced, at least approximately, then we would have

$$
\eta\left(\rho_{h}\right) \approx N_{m}^{1 / 2} \eta_{T}\left(\rho_{h}\right),
$$


where $N_{m}$ is the number of triangular finite elements in $\mathcal{T}_{\Omega}^{m}$. We thus mark an element $T \in T_{\Omega}^{m}$ for coarsening, if

$$
\eta_{T}\left(\rho_{h}\right) \leq \theta \frac{\eta\left(\rho_{h}\right)}{N_{m}^{1 / 2}}
$$

with some $\theta \in(0,1)$. Notice that these estimators are used only for the coarsening criterion. Notice also that we do not refine the time step adaptively.

\subsection{Adaptivity for boundary evolution}

A simple adaptive strategy is used for the boundary evolution. The 1d finite element mesh for the initial boundaries consists of elements that have almost a uniform element size. This size is kept approximately constant during the time evolution. Nodes are inserted in or removed from the mesh in each time step according to the criterion that the distance between neighboring nodes is almost a constant. Such an adaptive method is efficient and accurate as long as the boundary curvature is not too large. If the curvature will be large other refinement methods can be used.

\subsection{Element marking}

In the weak formulation (3.2) for the adatom diffusion, the extended diffusion constant $D_{i}$, the extended deposition flux rate $F_{i}$, and the extended desorption rate $\tau_{i}^{-1}$ are piecewise constant functions. Here, we drop the time-step index $m$. They are discontinuous across the boundaries $\Gamma_{i, h}$ and $\Gamma_{i+1, h}$. Thus, to track the value of such piecewise constant functions, we need to mark each element to track the information whether this element lies entirely in $\Omega_{i}$ or it is crossed by a boundary $\Gamma_{i, h}$. In addition, such element marking can keep track of the heights of terraces $\Omega_{i}(t)(0 \leq i \leq N)$.

To proceed, we use the orientation of the boundaries $\Gamma_{i, h}$ to generate the marking of the initial $2 \mathrm{~d}$ finite element triangulation, cf. Figure 3 (left), where all the inner elements belong to the upper terrace and all the outer elements belong to the lower terrace, cf. Figure 3 (right). This information is tracked through the simulation. Using a marker $\operatorname{mark}(T)$, we mark every element $T$ by

$$
\operatorname{mark}(T)=\left\{\begin{array}{ll}
i & \text { if } T \subseteq \overline{\Omega_{i}} \\
i+1 / 2 & \text { if } \operatorname{int}(T) \cap \Gamma_{i+1, h} \neq \emptyset
\end{array},\right.
$$

where $\operatorname{int}(T)$ denotes the interior of $T$. We assume:

(A) for every element $T$, there is at most one $i$ such that $\operatorname{int}(T) \cap \Gamma_{i, h} \neq \emptyset$;

(B) for every terrace $\Omega_{i}$, there is at least one element $T$ such that $T \subseteq \overline{\Omega_{i}}$;

(C) any element with mark $i+1 / 2$ is adjacent to exactly one element with mark $i$ or one element with mark $i+1$.

By assumptions (A) and (B), the marking of all elements is well-defined for sufficiently fine meshes. Assumption (C) can always be satisfied by moving the intersection point of the $1 \mathrm{~d}$ and $2 \mathrm{~d}$ mesh, if it lies on a $2 \mathrm{~d}$ node or two subsequent intersection points lie on the same $2 \mathrm{~d}$ edge. The moving is only done virtually. Note that assumption $(\mathrm{C})$ implies 

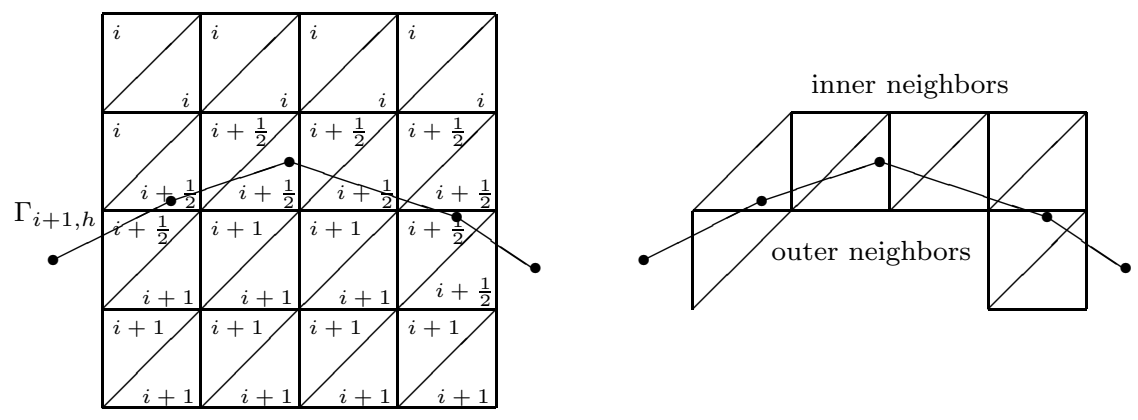

Figure 3: Marking of elements $T$ in a triangulation $\mathcal{T}_{h}$ near a boundary $\Gamma_{i+1, h}$.

that each element marked by $i+1 / 2$ has either one inner or one outer neighbor, marked by $i+1$ or $i$, respectively, cf. Figure 3 (right).

In each time step, the marking changes according to the evolution of the moving boundaries $\Gamma_{i, h}$. During refinement, the marking is passed form parent elements to child elements. The marking of elements obtained by coarsening is reset to -1 . It is calculated in the next time step again using the marking of the neighboring elements. With this strategy, the information of the terrace height can be tracked. The piecewise constants $D_{i}, F_{i}$ and $\tau_{i}^{-1}$ are now well defined due to the marking of the elements. In addition, for elements marked by $i+1 / 2$, the orientation of $\Gamma_{i+1, h}$ indicates which part of the element belongs to $\Omega_{i}$ and which part to $\Omega_{i+1}$.

Marking algorithm. Start with the initial triangulation $\mathcal{T}_{\Omega}^{0}$ and the initial boundary $\Gamma_{i+1, h}^{0}$. Set $m=0$.

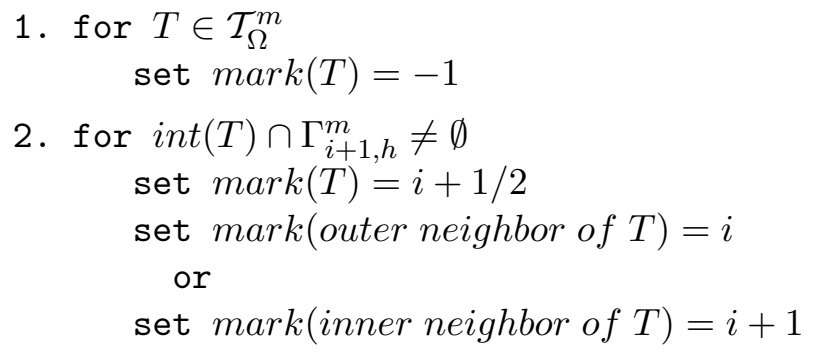

3. while $\exists T$ s.t. $\operatorname{mark}(T)=-1$

for $T \in \mathcal{T}_{\Omega}^{m}$

if $\operatorname{mark}(T)=-1$ and $\operatorname{mark}($ neighbor of $T) \neq-1$

set $\operatorname{mark}(T)=\operatorname{mark}($ neighbor of $T)$

4. perform time step, adapt mesh, set $m=m+1$

5. for $T \in \mathcal{T}_{\Omega}^{m}$

if $\operatorname{mark}(T)=i+1 / 2$

reset $\operatorname{mark}(T)=-1$

6. go to 2

With this algorithm, each element in the initial mesh is marked. However, in each time step, marks change only for elements $T$ with $\operatorname{int}(T) \cap \Gamma_{i, h}^{m} \neq \emptyset$, and elements $T$ 
whose marks are reset to -1 due to coarsening or $\operatorname{int}(T) \cap \Gamma_{i, h}^{m-1} \neq \emptyset$. To illustrate our method of marking, we show in Figure 4 a refined $2 \mathrm{~d}$ mesh, a 1d mesh of a boundary, and the marking of the elements, for which different colors or grey scales represent different markers. The element marking can be viewed as a discrete height function for the growing film.
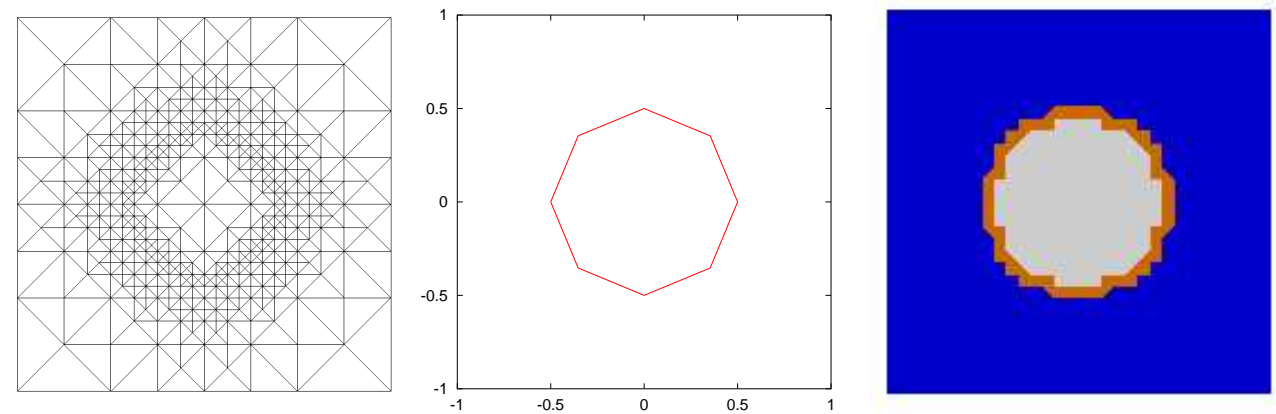

Figure 4: A refined 2d mesh, a 1d mesh of a boundary, and element marking.

\subsection{Numerical integration}

The assembly of the finite element system for Problem 3.2 involves several nonstandard integrals. One class of such integrals are those involving coefficients such as $D_{i}, F_{i}$, and $\tau_{i}^{-1}$ that are discontinuous within one element. Here, again, we drop the index $m$. Another class of such integrals are the boundary integrals that appear in the diffusion equation, coupling the adatom density and the moving boundary.

Let us first treat the integrals involving discontinuous coefficients. We need to evaluate the integrals

$$
\int_{T} D_{i} \nabla \phi_{k} \cdot \nabla \phi_{l}, \quad \int_{T} D_{i-1} \nabla \phi_{k} \cdot \nabla \phi_{l}, \quad \int_{T} F_{i} \phi_{l}, \quad \int_{T} F_{i-1} \phi_{l}, \quad \int_{T} \tau_{i}^{-1} \phi_{l}, \quad \int_{T} \tau_{i-1}^{-1} \phi_{l}
$$

over an element $T$ with $\operatorname{int}(T) \cap \Gamma_{i, h} \neq \emptyset$. These integrals are of the form $\int \phi \lambda$ with $\phi$ a smooth function and $\lambda$ a discontinuous function,

$$
\lambda= \begin{cases}\lambda_{i-1} & \text { in } T \cap \overline{\Omega_{i-1}} \\ \lambda_{i} & \text { in } T \cap \overline{\Omega_{i}}\end{cases}
$$

with some $\lambda_{i-1}, \lambda_{i} \in \mathbb{R}$, cf. Figure 5 . We use the following integral approximation due to [39], cf. Figure 5:

$$
\begin{aligned}
\int_{T} \lambda \phi & \approx \int_{\triangle(D B E)} \lambda_{i} \phi+\int_{\square(A D E C)} \lambda_{i-1} \phi \\
& =\int_{\triangle(D B E)} \lambda_{i} \phi+\int_{T} \lambda_{i-1} \phi-\int_{\triangle(D B E)} \lambda_{i-1} \phi .
\end{aligned}
$$

Note that this formula avoids the explicit integration over quadrilaterals and requires only integration over triangles, and can be thus performed in a nearly standard way. 

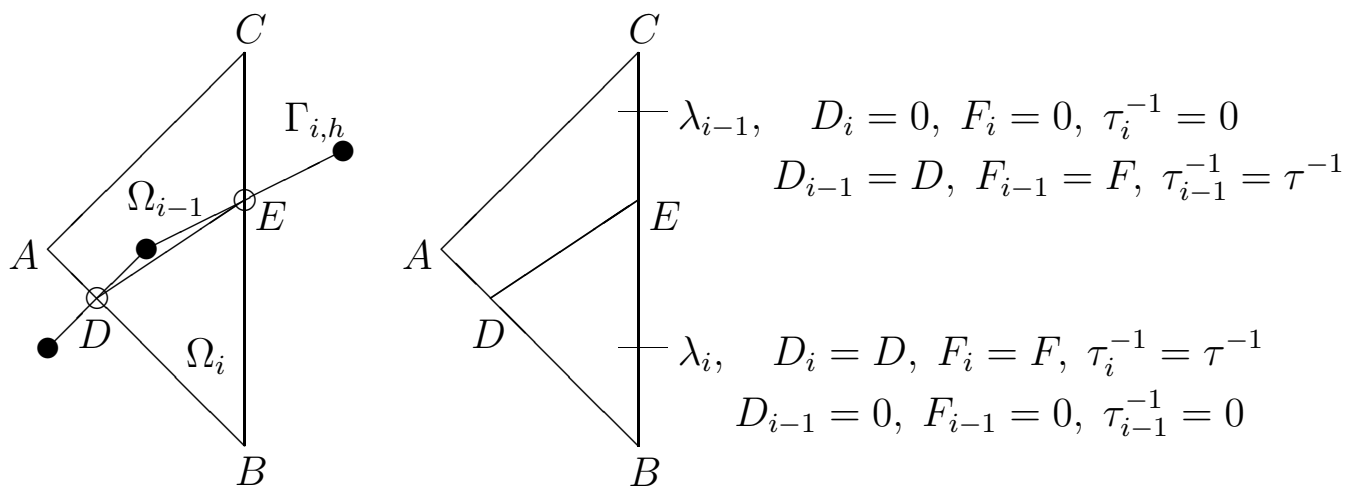

Figure 5: Element $T$, boundary $\Gamma_{i, h}$, and definition of $\lambda, D_{i}, F_{i}$, and $\tau_{i}^{-1}$.

The line integration over a boundary $\Gamma_{i, h}$ in the adatom diffusion problem is treated by subdividing the boundary into polygons, see Figure 6 . A polygon is defined by the intersection points of $\Gamma_{i, h}$ and boundaries of the element $T$, and points of $\Gamma_{i, h}$ where the parameterization changes. The integration can then be performed in a standard way by calculating integrals of piecewise linear functions. See [34] for further details.

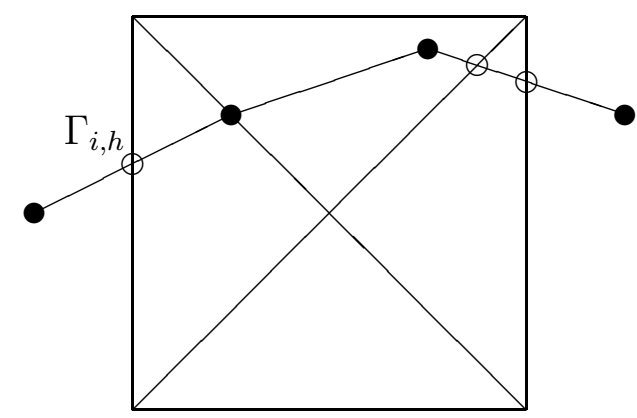

Figure 6: Element $T$, boundary $\Gamma_{i, h}$, and line integration path.

\subsection{Algorithm}

Combining Algorithm 3.1 for the adatom diffusion and Algorithms 3.2 and 3.3 for the boundary evolution, as well as the routines described in Sections 4.1-4.4, we arrive at the following algorithm:

Algorithm 4.1 Let $\rho_{i, h}^{0}, \Gamma_{i, h}^{0}$ and $\Omega_{i}^{0}$ be given. Mark all elements according to their positions related to $\Omega_{i}^{0}$. Define $D_{i}, F_{i}$ and $\tau_{i}^{-1}$. Set $m=0$.

1. compute boundaries $\Gamma_{i, h}^{m+1}$ and curvatures $\kappa_{i, h}^{m+1}$ 
(a) compute $v_{i, h}^{m+1}, \vec{v}_{i, h}^{m+1}$, and $\Gamma_{i, h}^{m+1}$

(b) refine and coarse $\Gamma_{i, h}^{m+1}$

(c) compute $\kappa_{i, h}^{m+1}$ on $\Gamma_{i, h}^{m+1}$

2. compute adatom densities $\rho_{i, h}^{m+1}$

(a) refine and coarse $\mathcal{T}_{h}^{m}$

(b) update marking of elements and definition of $D_{i}, F_{i}$ and $\tau_{i}^{-1}$

(c) compute $\rho_{i, h}^{m+1}$

(d) compute $\gamma_{i}=\gamma_{i}\left(\rho_{i, h}^{m+1}, \rho_{i-1, h}^{m+1}\right)$

3. set $m:=m+1$, go to 1

\section{$5 \quad$ Numerical results}

We first present numerical results on the geometric motion of curves in Section 5.1 and on mass balance and conservation of area in Section 5.2. We then, in Section 5.3, investigate numerically the growth of a single circular island, and compare the numerical results with the known analytical solutions. Finally, in Section 5.4, we apply our numerical algorithm to simulate the growth of a non-circular island, and compare the numerical results for the case with and without surface diffusion.

Unless otherwise stated, we use the following data in all the numerical simulations:

- Parameters: $D=10^{5}, F=1, \rho^{*}=10^{-5}, k_{+}=k_{-}=10^{5}, \mu=1, \nu=10$;

- Domain: $\Omega$ is a circular domain with radius 3 ;

- Number of elements of the initial 1d finite element mesh: 128;

- Time step: $10^{-6}$ with surface diffusion, $10^{-4}$ without surface diffusion.

The unit of length is the substrate lattice spacing. Thus the deposition rate $F$ denotes the number of atoms deposited per unit time and adsorption site and $D$ is the "hopping rate".

\subsection{Geometric motion of curves}

Our first test example is a purely geometric motion of curves governed by Problem 3.3 in Section 3.2, decoupled from the adatom diffusion. Choosing the parameters $\alpha, \beta$ and $\gamma_{i}$ in a suitable way, we apply the algorithm to the following geometric evolution equations:

Case 1: mean curvature flow: $\alpha=0, \beta=1$ and $\gamma_{i}=0$;

Case 2: surface diffusion: $\alpha=1, \beta=0$ and $\gamma_{i}=0$;

Case 3: surface diffusion and mean curvature flow: $\alpha=1, \beta=1$ and $\gamma_{i}=0$. 
Rectangle. Starting with a rectangle as initial curve, we see that all three geometric motions will smooth the curve to a circle. Figure 7 shows 8 snapshots of the evolution for each of the three cases. The final times are chosen as the time where the circular shape is reached. As expected, surface diffusion is area preserving, and mean curvature flow is curve shortening, thus area shrinking. In Case 3, surface diffusion dominates the smoothing due to its much faster time scale.

Perturbed circle. A further test on the evolution of a perturbed circle is captured in Figure 8 . The perturbation is a superposition of sines:

$$
\delta(\theta)=0.05 \sin (3 \theta)+0.1 \sin (12 \theta),
$$

where $\theta$ is the azimuthal angle. As expected, in all three cases, perturbations are smoothed out and high frequencies are damped faster than low frequencies. But the time-scales for mean curvature flow and surface diffusion are quite different. In Case 1 (mean curvature flow), the time elapsed until the high frequencies are completely damped is $2.0 \times 10^{-2}$, whereas the time for the decay of the low frequency waves is 0.2 . In Case 2 (surface diffusion), the high frequency waves are damped already after $5.0 \times 10^{-4}$, whereas a circle appears at $5.0 \times 10^{-2}$. The difference of the time-scales for the damping of high and low frequencies is about 10 for mean curvature and 100 for surface diffusion. This is related to the 4 th order operator of surface diffusion. Due to these different time scales, surface diffusion dominates the smoothing in Case 3. This is why there is no qualitative difference between Case 2 and Case 3 (despite the shrinking of the area). Again the final times are chosen as the time where a circular shape is reached.

\subsection{Area conservation and mass balance}

We now test our numerical method for the area conservation and mass balance. To this end, we exclude the desorption and set the attachment rates to be zero: $k_{+}=k_{-}=0$. Thus, the fluxes $q_{i}^{+}$and $q_{i}^{-}$to the island boundaries are zero. Consequently, the area of the island should not increase. In fact, in this case we have for any $i$ with $0 \leq i \leq N$,

$$
\begin{aligned}
\frac{d}{d t}\left|\Omega_{i}(t)\right| & =\frac{d}{d t} \int_{\Omega_{i}(t)} 1=\int_{\Gamma_{i}(t)} v_{i}-\int_{\Gamma_{i+1}(t)} v_{i+1} \\
& =\int_{\Gamma_{i}(t)} \nu \partial_{s s} \kappa_{i}-\int_{\Gamma_{i+1}(t)} \nu \partial_{s s} \kappa_{i+1}=0,
\end{aligned}
$$

where obvious modifications are needed for the case $i=0$ and $i=N$. This shows that the area of all islands of the same height $i$ should be a constant. Moreover, the mass of the islands of height $i$ should increase linearly due to the deposition of material with a constant flux rate $F$. Indeed, observing that $q_{i}^{+}=q_{i}^{-}=0$ for all $i=0, \ldots, N$, and using 
Case 1: mean curvature flow

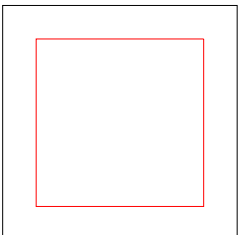

$t=0.0$

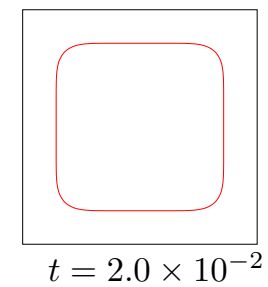

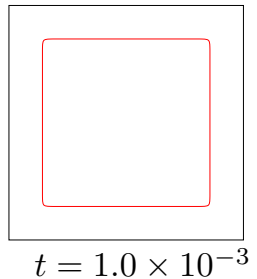

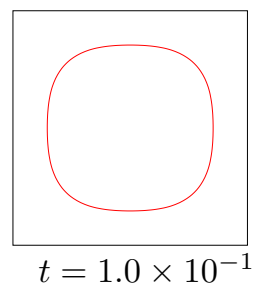

Case 2: surface diffusion

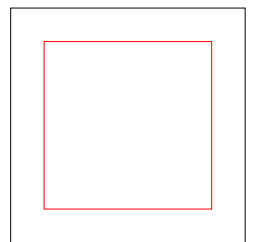

$t=0.0$

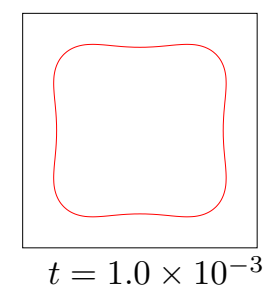

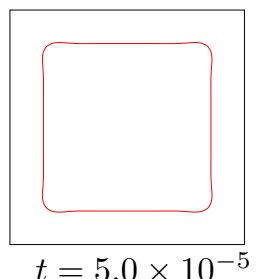

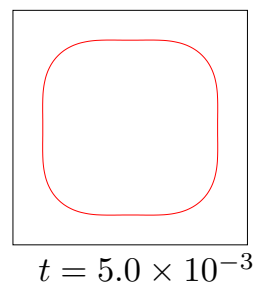

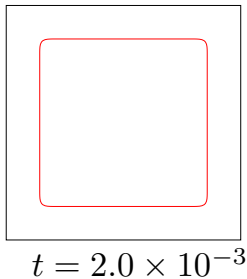
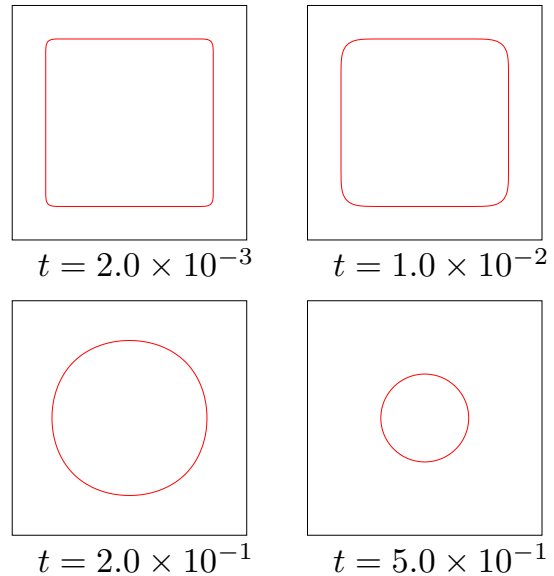

Case 3: mean curvature and surface diffusion

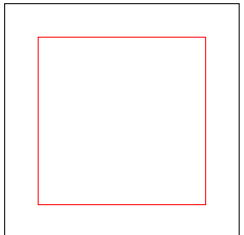

$t=0.0$

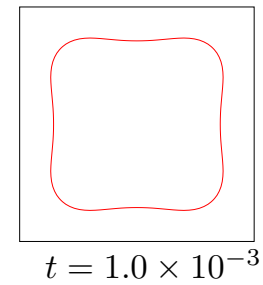

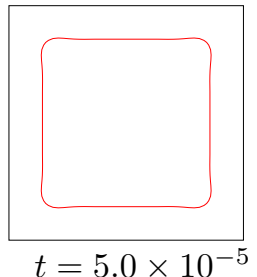

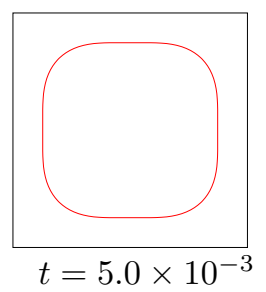

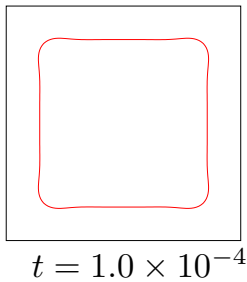
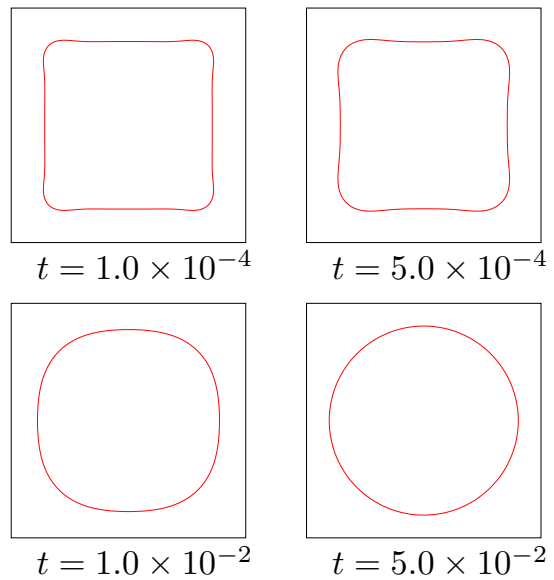

Figure 7: Geometric motion of a rectangle. 
Case 1: mean curvature flow

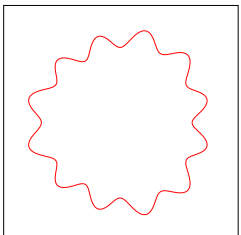

$t=0.0$

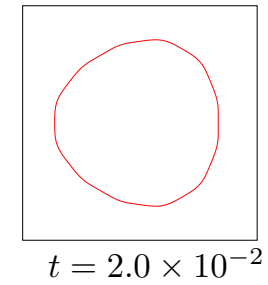

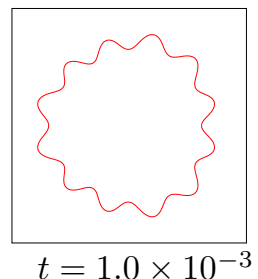

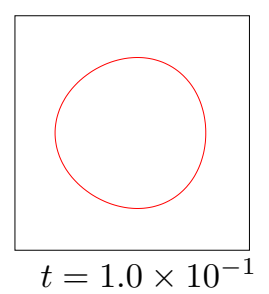

Case 2: surface diffusion

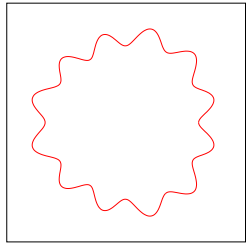

$t=0.0$

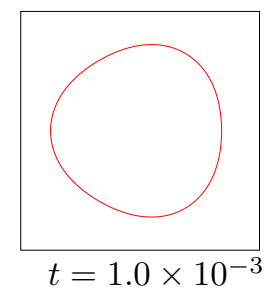

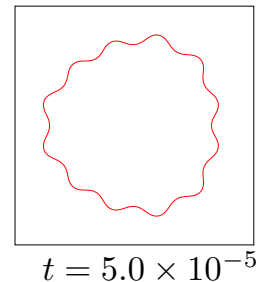

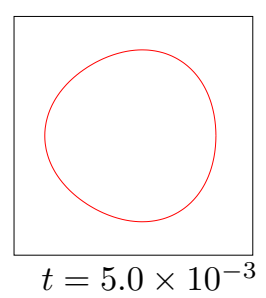

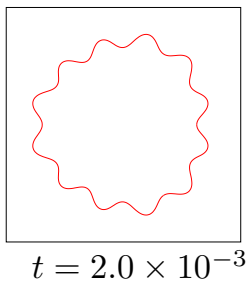
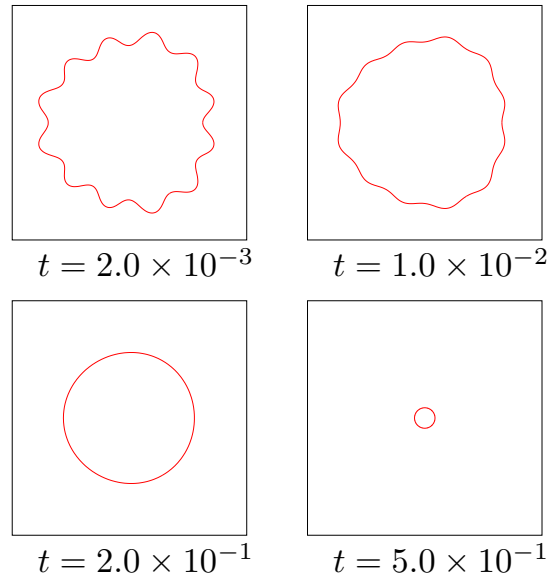

Case 3: mean curvature and surface diffusion

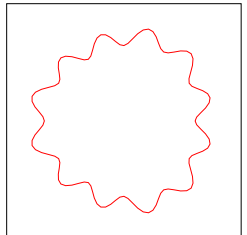

$t=0.0$

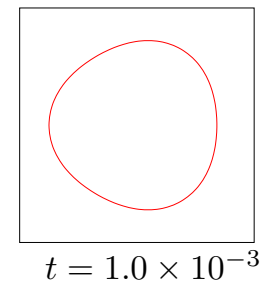

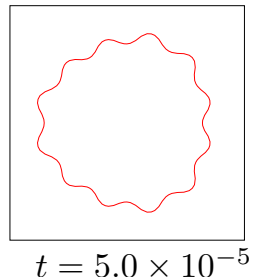

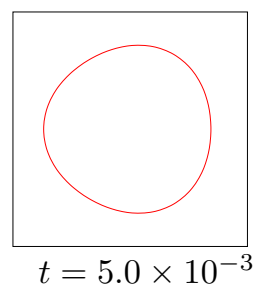

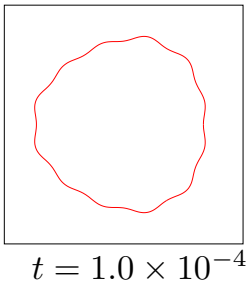
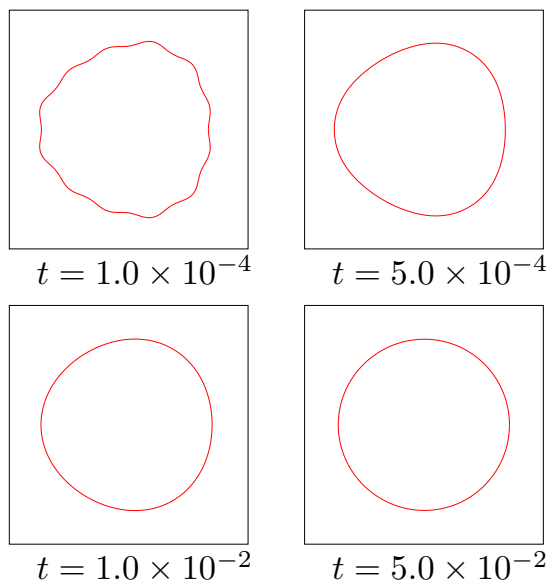

Figure 8: Geometric motion of a perturbed circle. 
$(2.2)-(2.4)$ and (5.1), we obtain

$$
\begin{aligned}
\frac{d}{d t} & \int_{\Omega_{i}(t)} \rho_{i}(t)=\int_{\Omega_{i}} \frac{\partial \rho_{i}}{\partial t}+\int_{\Gamma_{i}} v_{i} \rho_{i}-\int_{\Gamma_{i+1}} v_{i+1} \rho_{i} \\
& =\int_{\Omega_{i}}\left(D \Delta \rho_{i}+F\right)+\int_{\Gamma_{i}} v_{i} \rho_{i}-\int_{\Gamma_{i+1}} v_{i+1} \rho_{i} \\
& =F\left|\Omega_{i}\right|+\int_{\Gamma_{i}} D \nabla \rho_{i} \cdot \vec{n}_{i}-\int_{\Gamma_{i+1}} D \nabla \rho_{i} \cdot \vec{n}_{i+1}+\int_{\Gamma_{i}} v_{i} \rho_{i}-\int_{\Gamma_{i+1}} v_{i+1} \rho_{i} \\
& =F\left|\Omega_{i}\right|=F\left|\Omega_{i}(0)\right|
\end{aligned}
$$

Therefore, the mass on the islands of height $i$ is

$$
\int_{\Omega_{i}(t)} \rho_{i}(t)=F\left|\Omega_{i}(0)\right| t+\int_{\Omega_{i}(0)} \rho_{i}(0) .
$$

Furthermore, due to the no-flux boundary condition on $\partial \Omega$, the mass in the whole system increases linearly

$$
\int_{\Omega} \rho(t)=F|\Omega| t+\int_{\Omega} \rho(0) .
$$

Under the assumptions made in this section, we numerically compute the area and mass at different times for a single, growing island $(N=1)$. We consider two different initial configurations $\Omega_{1}(0)$ : (a) a circular island of radius 1 and (b) a perturbed circular island of radius 1 with perturbation

$$
\delta(\theta)=0.05 \sin (3 \theta)+0.1 \sin (12 \theta) .
$$

Area conservation. Figure 9 shows the simulation of the evolution of the perturbed island boundary at various times and the computed area at these times.

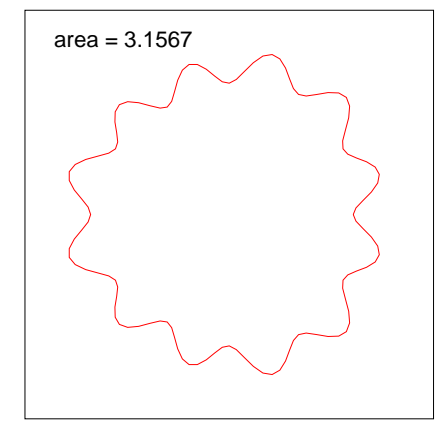

$t=0.0$

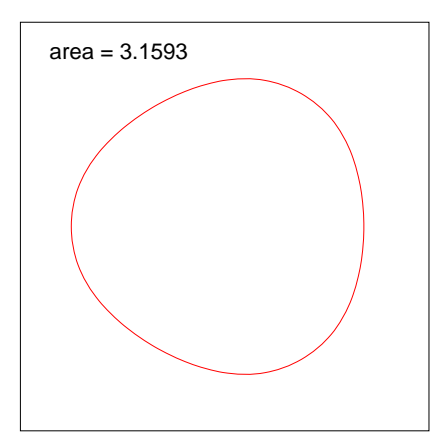

$t=5.0 \times 10^{-5}$

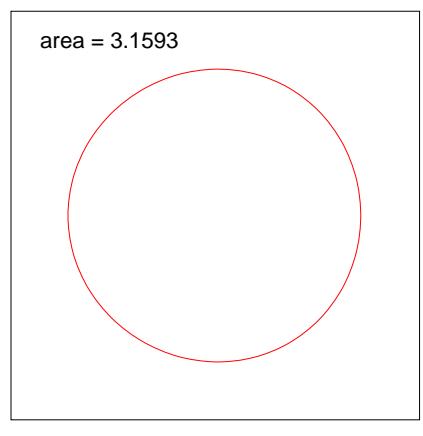

$t=3.0 \times 10^{-3}$

Figure 9: Conservation of the island area in the case $k_{+}=k_{-}=0$.

The area is conserved to a very satisfactory extent: during the time period in which the island smoothes to a circular island, the area change is less than $0.1 \%$. In the case of a circular island the area is exactly conserved. 
Mass balance. Using the previously derived formulas (5.2) and (5.3), we expect the mass corresponding to the circular island to be

$$
\int_{\Omega_{1}(t)} \rho_{i}(t) \approx 3.14 t+0.0000314
$$

and the mass in the whole system to be

$$
\int_{\Omega} \rho(t) \approx 28.3 t+0.000283
$$

Figure 10 shows the computed mass for both cases: the circle and the perturbed circle, where the functions $f$ and $g$ are the least-squares fits of the data to affine functions. The fitted parameters for the growth rate of mass in both cases are in good agreement with the analytical results. The relative error is $0.8 \%$ and $1.2 \%$ for the circular and the perturbed circular island, respectively.
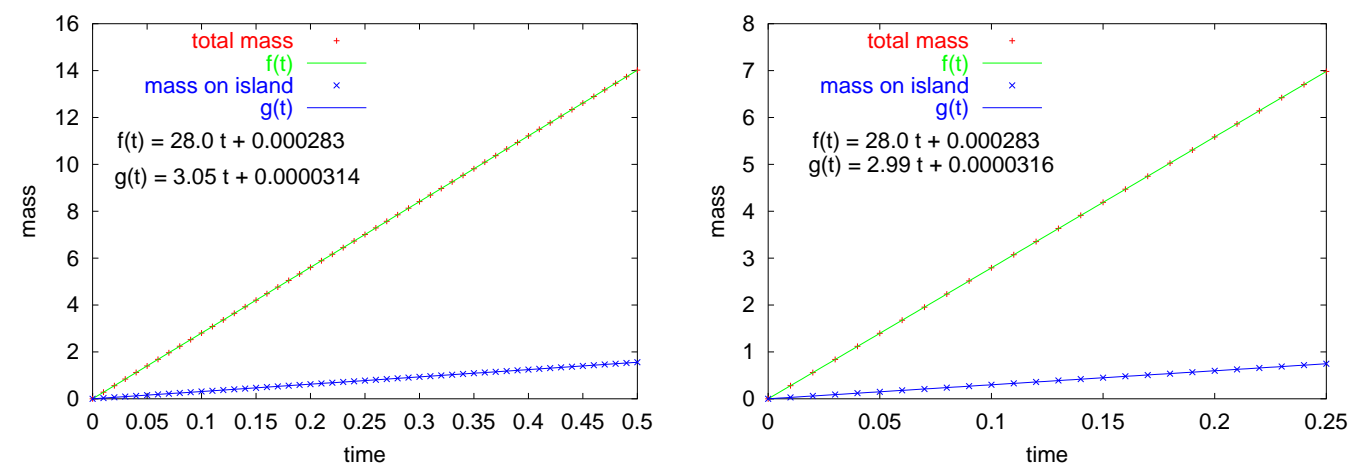

Figure 10: Mass increase: circular island (left) and perturbed circular island (right).

\subsection{Growth of a single circular island}

We now apply our numerical method to the case of a single, growing circular island and compare the computational results with the analytic solution of a quasi-stationary approximation. For the purpose of testing, we assume that there is no desorption.

Consider a single, circular island $\Omega_{1}(t)$ of radius $R(t)$ at time $t$ that is growing on a terrace which is a concentric circular region with radius $R_{\Omega}$. In the quasi-stationary approximation of the adatom diffusion, the time dependence in the diffusion equation is dropped. This approximation is valid if $F / D \ll 1$. In [23], an analytic solution is derived under this assumption. With a set of parameters satisfying $F / D \ll 1$, we expect our simulation of the time dependent diffusion equation to be in good agreement with the analytic solution of the quasi-stationary diffusion equation.

Using polar coordinates $(r, \theta)$ with the origin at the center of the circular island, the 
radially symmetric solution of the quasi-stationary diffusion equation is given by [23]

$$
\begin{aligned}
& \rho_{0}(r, t)=\frac{F}{4 D}\left(R(t)^{2}-r^{2}\right)+\frac{F R_{\Omega}^{2}}{2 D} \ln \left(\frac{r}{R(t)}\right)+\rho^{*}\left(1+\frac{\mu}{R(t)}\right)+\frac{F}{2 k_{-}}\left(\frac{R_{\Omega}^{2}}{R(t)}-R(t)\right), \\
& \rho_{1}(r, t)=\frac{F}{4 D}\left(R(t)^{2}-r^{2}\right)+\rho^{*}\left(1+\frac{\mu}{R(t)}\right)+\frac{F R(t)}{2 k_{+}} .
\end{aligned}
$$

Since the curvature $\kappa_{1}=1 / R(t)$ of the circular boundary $\Gamma_{1}(t)$ is spatially constant, we have $\partial_{s s} \kappa_{1}=0$. Moreover, since the velocity of the circular boundary $\Gamma_{1}(t)$ is given by $v_{1}=R^{\prime}(t)$, we obtain by a simple calculation that $R^{\prime}(t)=F R_{\Omega}^{2} /(2 R(t))$, i.e., $\left(R(t)^{2}\right)^{\prime}=$ $F R_{\Omega}^{2}$. Thus, we obtain the dynamic law

$$
R(t)^{2}=F R_{\Omega}^{2} t+R(0)^{2}
$$

for the evolution of the circular boundary $\Gamma_{1}(t)$. Furthermore, at the island boundary $\Gamma_{1}(t)$, we have

$$
\rho_{1}(t)-\rho_{0}(t)=\frac{F}{2} R(t)\left(\frac{1}{k_{+}}+\frac{1}{k_{-}}-\frac{1}{k_{-}} \frac{R_{\Omega}^{2}}{R(t)^{2}}\right) .
$$

Thus, the adatom density $\rho$ is discontinuous at $\Gamma_{1}(t)$.

In the simulation the island remains a circle. Figure 11 shows the profile of the adatom density at various times. It also shows the growth rate of the island area, where again the function $f$ is the least-squares fit of the data to an affine function.
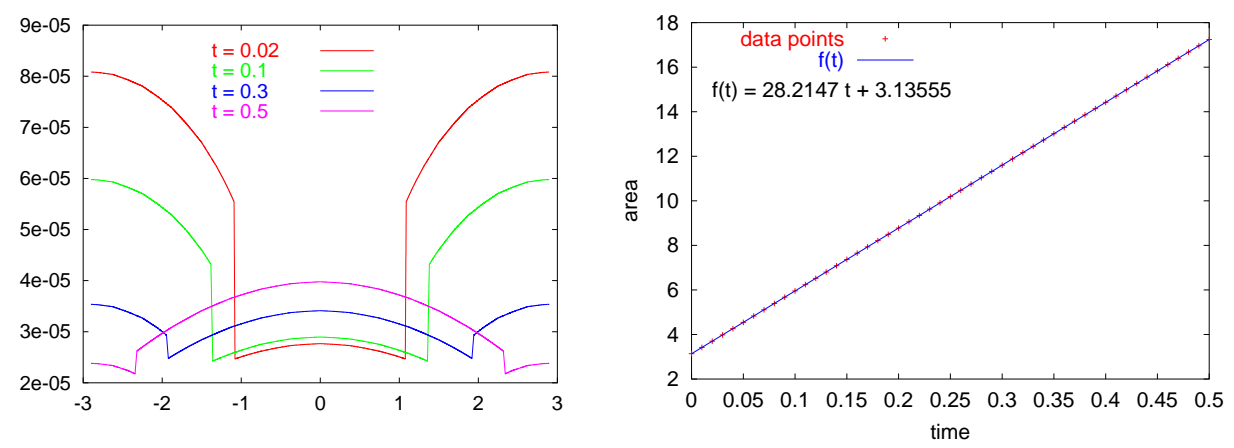

Figure 11: Adatom density profile (left) and area growth rate (right).

According to (5.4), one expects a growth rate of area $F|\Omega|=28.3$ as in Section 5.2. Our simulations are in good agreement with this value, see Figure 11 (right). The relative error is $0.2 \%$. In agreement with (5.5) the jump of the adatom density at the island boundary decreases with increasing area of the upper terrace, changing sign when both terraces exhibite the same area (since $k_{+}$equals $k_{-}$). Due to the jump in the adatom density max-errors between the numerical and analytical solution are only evaluated in $\Omega \backslash \delta \Gamma_{1}(t)$ with $\delta \Gamma_{1}(t)=\{x|R(t)-\delta<| x \mid<R(t)+\delta\}$ and $\delta=2 \cdot 10^{-2}$. The values are shown in Table 1. 


\begin{tabular}{|l|l|l|l|l|}
\hline time & 0.02 & 0.1 & 0.3 & 0.5 \\
\hline max-error & $6 \cdot 10^{-3}$ & $7 \cdot 10^{-3}$ & $9 \cdot 10^{-3}$ & $8 \cdot 10^{-3}$ \\
\hline
\end{tabular}

Table 1: Max-errors between numerical and analytical solution.

\subsection{Smoothing properties and more islands}

We apply the numerical method to show the influence of one-dimensional "surface" diffusion on the smoothing of island boundaries. We study a single growing island evolving from an initially perturbed circular island.
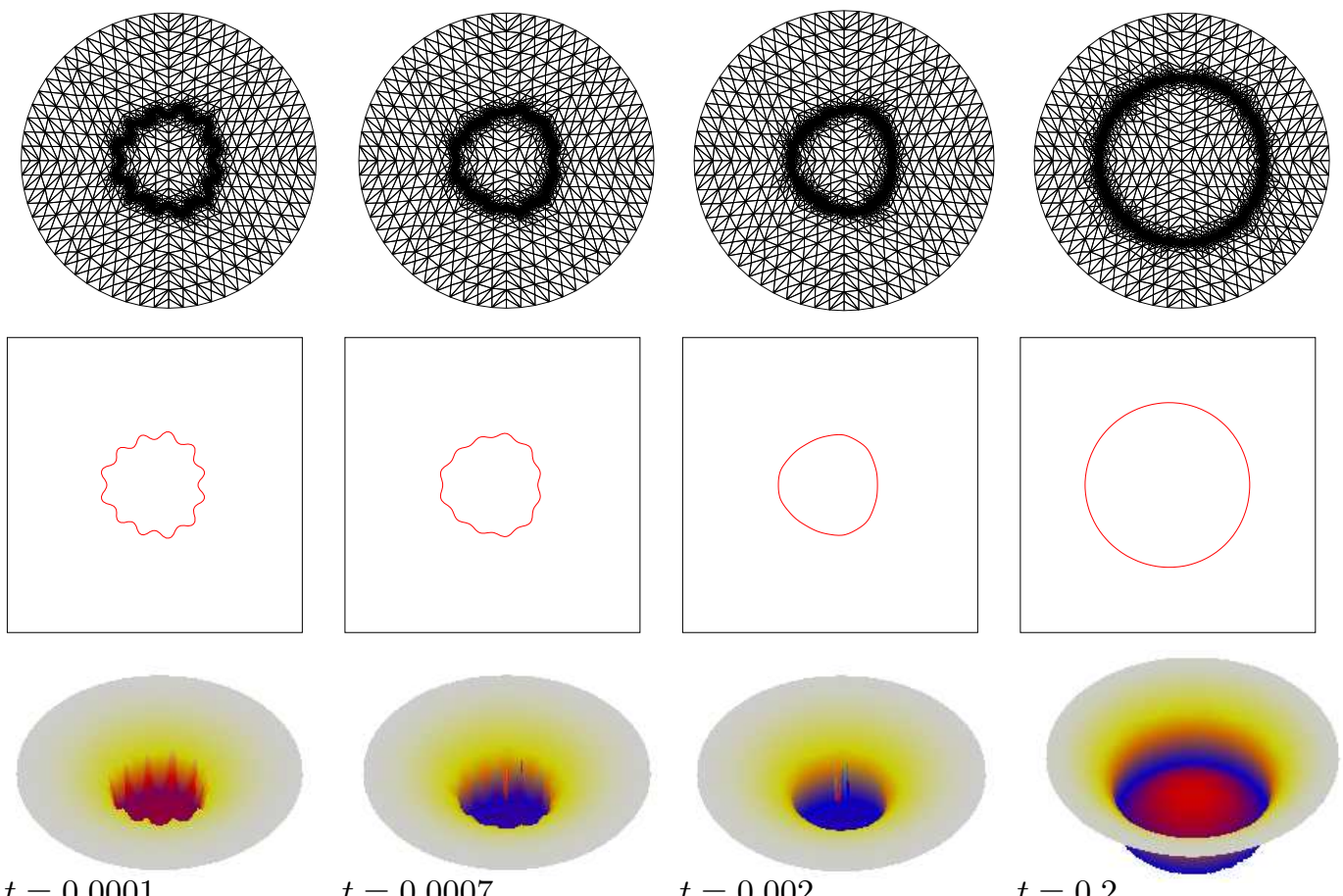

$t=0.0001$

$t=0.0007$

$t=0.002$

$t=0.2$

Figure 12: 2d mesh, 1d boundary and adatom density: with surface diffusion.

The perturbation is again a superposition of sines:

$$
\delta(\theta)=0.05 \sin (3 \theta)+0.1 \sin (12 \theta) .
$$

We compare results for two different values of $\nu: \nu=0.1$ and $\nu=0$. From the analysis in [23], we expect the high frequencies to be rapidly damped, whereas the amplitude of the low frequency waves should decay slowly, as in the pure geometrical case in Section 5.1. Furthermore, in the case with surface diffusion, the damping of the high frequencies should be much faster. 
Our numerical results, shown in Figure 12 and Figure 13, display the adaptively refined $2 \mathrm{~d}$ mesh, the computed $1 \mathrm{~d}$ boundary $\Gamma_{i, h}$, and the computed adatom density $\rho_{h}$ at various time instants for the case with and without surface diffusion, respectively.
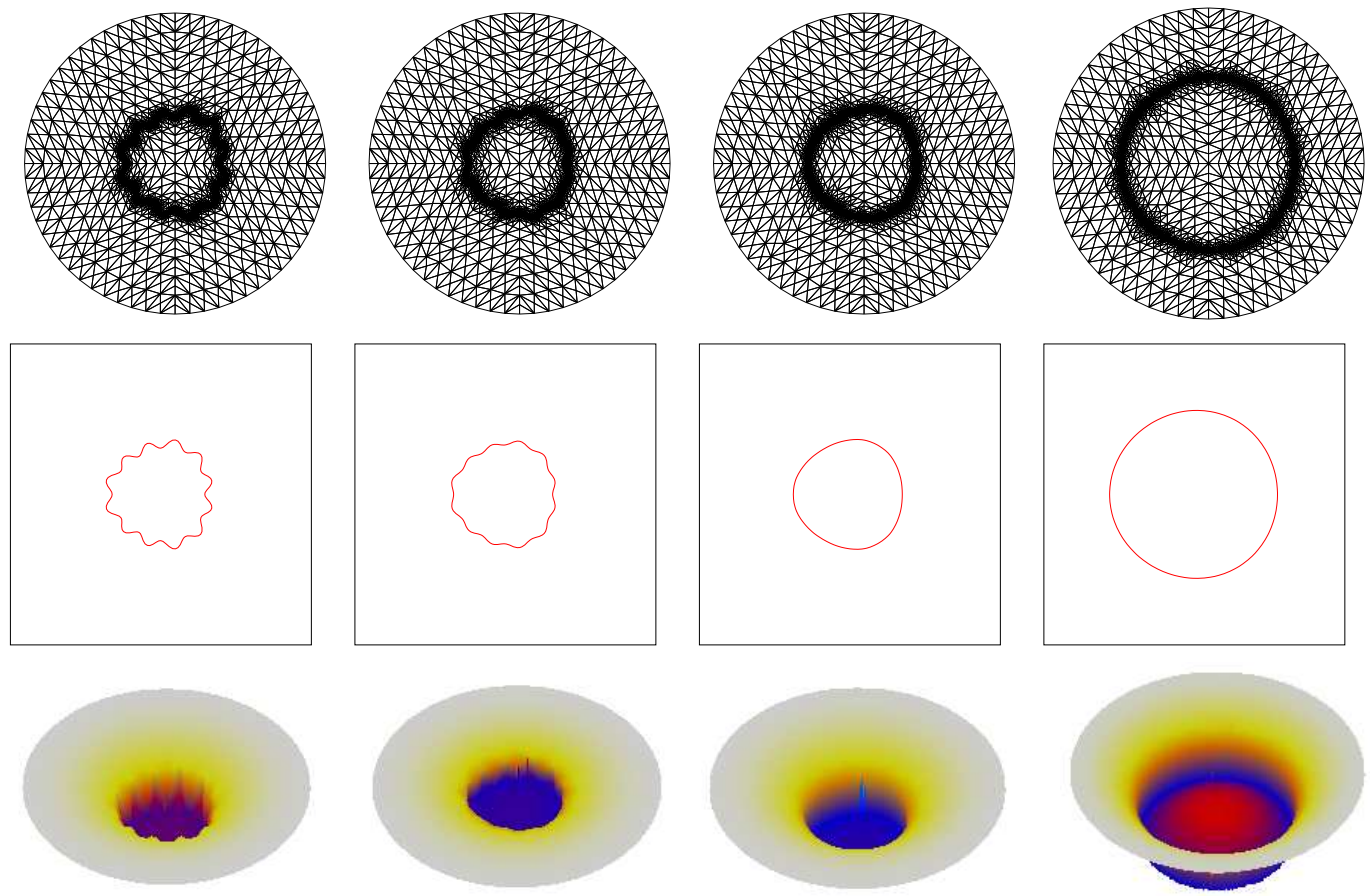

$t=0.001$

$t=0.005$

$t=0.02$

$t=0.2$

Figure 13: 2d mesh, 1d boundary and adatom density: without surface diffusion.

Comparing the two simulations, we see that the influence of surface diffusion is quite similar to that in the pure geometric setting of Section 5.1. The decay of the high frequency perturbation is accelerated approximately by a factor of 10 . Note that we have chosen the surface diffusion coefficient smaller than in Section $5.1(\nu=0.1$ instead of $\nu=1.0)$.

The growth rate of the islands in the simulations is approximately constant, in agreement with the expected value we compute $f(t)=28.21$ in both cases, which is a relative error of $0.2 \%$.

The final example shows the evolution of three islands. The marking of the elements, representing the height of the islands and the adatom density is shown in Figure 14.

\section{Conclusions}

In this work, we have developed an adaptive finite element method for the simulation of island dynamics in epitaxial growth of thin films. Our model is a free or moving boundary type problem that consists of the diffusion equation for the adatom density and the boundary evolution equation that determines the normal velocity of the moving boundaries. We focus on two physical mechanisms: the attachment-detachment kinetics 

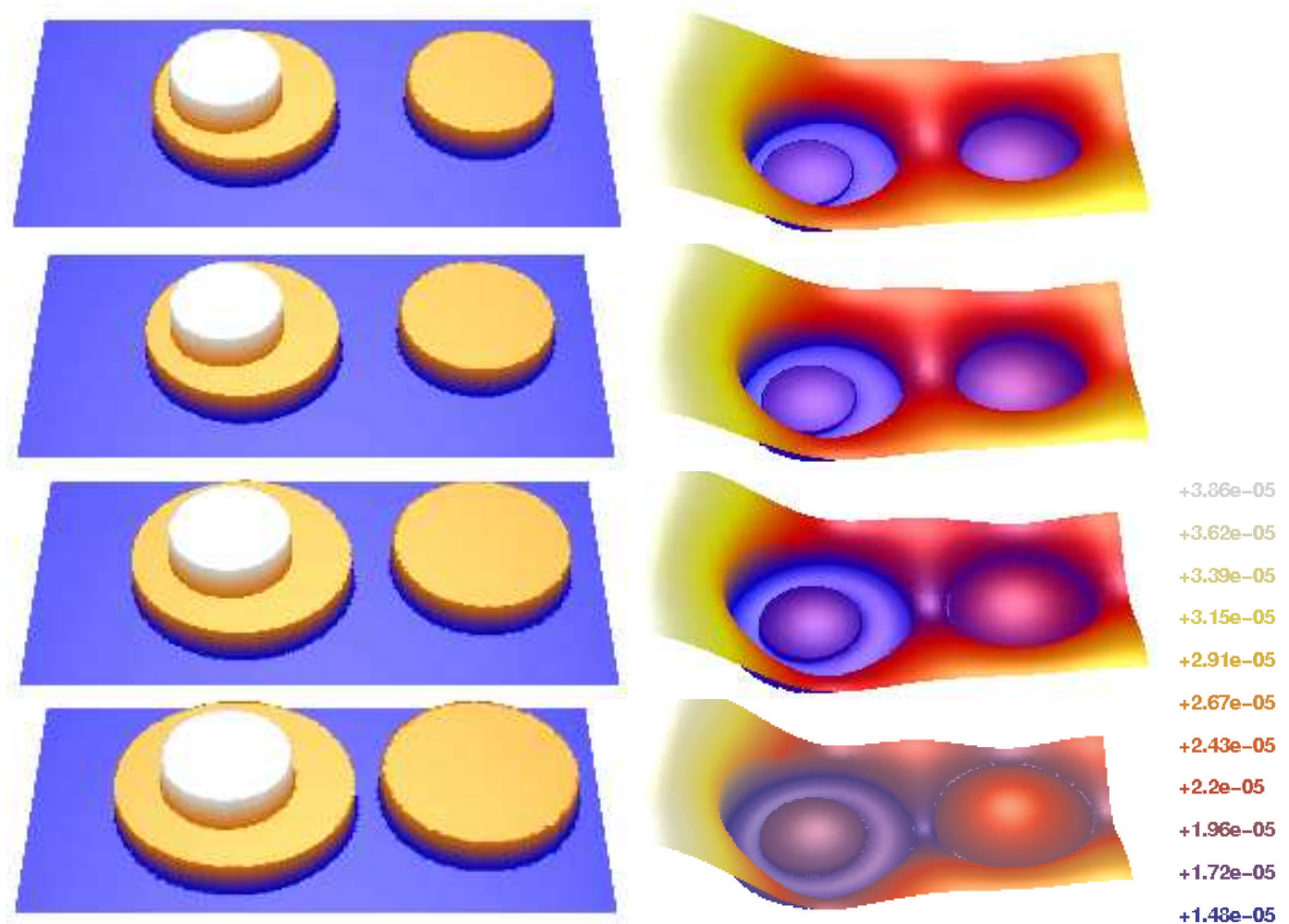

$+1.48 e-05$

Figure 14: Discrete height function and adatom density at various times.

that is modeled by a two-sided (from upper and lower terraces) Robin type boundary condition for the adatom density on moving boundaries; and the one-dimensional "surface" diffusion that is modeled by the one-dimensional "surface" Laplacian of curvature.

To treat the numerical difficulties that arise from modeling the two physical mechanisms, we have developed a technique of extension for the adatom diffusion and parametric, finite element, front-tracking method for the boundary evolution. We have also implemented adaptivity, element marking, and numerical integration on "irregular" elements.

Upon testing on different problems, we find that our method is stable, efficient, and fairly accurate. This method can be used for simulating island dynamics, in the absence of island nucleation and coalescence, for a relatively long period of time.

Acknowledgment. We would like to thank Pedro Morin for his assistance in implementing the algorithm for surface diffusion using parametric finite elements in ALBERT and Simon Vey for his help in implementing the coupling via TCP/IP. We would also like to thank Michael Griebel for his critical comments on the coupling and the adaptive strategy, and Michael Moske and Felix Otto for helpful discussions. The visit of Eberhard Bänsch, Omar Lakkis, and Bo Li to the Research Center caesar in Bonn during this research was supported by DFG through SFB 611. Bo Li was also partially supported by the NSF grant DMS-0072958. 


\section{References}

[1] G. S. Bales and A. Zangwill. Morphological instability of a terrace edge during step-flow growth. Phys. Rev. B, 41(9):5500-5508, 1990.

[2] E. Bänsch, P. Morin, and R. H. Nochetto. Finite element methods for surface diffusion. In Proceedings of International Conference on Free Boundary Problems: Theory and Applications, Trento, 2002 (to appear).

[3] E. Bänsch, P. Morin, and R. H. Nochetto. A finite element method for surface diffusion: The parametric case. In preparation, 2003.

[4] A.-L. Barabási and H. E. Stanley. Fractal Concepts in Surface Growth. Cambridge University Press, Cambridge, 1995.

[5] D. Braess. Finite elements: Theory, fast solvers, and applications in solid mechanics. Cambridge University Press, Cambridge, second edition, 2001. Translated from the 1992 German edition by Larry L. Schumaker.

[6] W. K. Burton, N. Cabrera, and F. C. Frank. The growth of crystals and the equilibrium of their surfaces. Phil. Trans. Roy. Soc. London Ser. A, 243(866):299-358, 1951.

[7] R. E. Caflisch, W. E, M. F. Gyure, B. Merriman, and C. Ratsch. Kinetic model for a step edge in epitaxial growth. Phys. Rev. E, 59(6):6879-6887, 1999.

[8] R. E. Caflisch, M. F. Gyure, B. Merriman, S. Osher, C. Ratsch, D. Vvedensky, and J. Zink. Island dynamics and the level set method for epitaxial growth. Appl. Math Lett., 12:13-22, 1999.

[9] R. E. Caflisch and B. Li. Analysis of island dynamics in epitaxial growth of thin films. Multiscale Model. Simul., 1(1):150-171, 2003.

[10] S. Chen, B. Merriman, M. Kang, R. E. Caflisch, C. Ratsch, L.-T. Cheng, M. Gyure, R. P. Fedkiw, C. Anderson, and S. Osher. Level set method for thin film epitaxial growth. J. Comput. Phys., 167:475-500, 2001.

[11] D. Chopp and J. Sethian. Motion by intrinsic Laplacian of curvature. Interfaces and Free boundaries, 1:107-123, 1999.

[12] G. Dziuk. An algorithm for evolutionary surfaces. Numer. Math., 58-6:603-611, 1991.

[13] G. Ehrlich and F. G. Hudda. Atomic view of surface diffusion: tungsten on tungsten. J. Chem. Phys., 44:1036-1099, 1966.

[14] R. Ghez, H. G. Cohen, and J. B. Keller. The stability of growing or evaporating crystals. J. Appl. Phys., 73:3685-3693, 1993.

[15] R. Ghez and S. S. Iyer. The kinetics of fast steps on crystal surfaces and its application to the molecular beam epitaxy of silicon. IBM J. Res. Develop., 32:804-818, 1988.

[16] M. F. Gyure, C. Ratsch, B. Merriman, R. E. Caflisch, S. Osher, J. Zinck, and D. Vvedensky. Level set method for the simulation of epitaxial phenomena. Phys. Rev. E, 58:R6931, 1998.

[17] M. A. Herman and H. Sitter. Molecular Beam Epitaxy: Fundamentals and Current Status, volume 7. Springer-Verlag, 2nd edition, 1997. 
[18] A. Karma and C. Misbah. Competition between noise and determinism in step flow growth. Phys. Rev. Lett., 71:3810-3813, 1993.

[19] J. B. Keller, H. G. Cohen, and G. J. Merchant. The stability of rapidly growing crystals. J. Appl. Phys., 73:3694-3697, 1993.

[20] S. V. Khare and T. L. Einstein. Unified view of step-edge kinetics and fluctuations. Phys. Rev. B, 57(8):4782-4797, 1998.

[21] M. Khenner, A. Averbuch, M. Israeli, and M. Nathan. Numerical simulation of grain boundary growing by level set methods. J. Comput. Phys., 170:764-784, 2001.

[22] J. Krug. Four lectures on the physics of crystal growth. Physica A, 318:47-82, 2002.

[23] B. Li, A. Rätz, and A. Voigt. Stability of a circular epitaxial island. Preprint No. 50, SFB 611, Universitt Bonn, 2002 (submitted).

[24] Z. Li, H. Zhao, and H. Gao. A numerical study of electro-migration voiding by evolving level set functions on a fixed Cartesion grid. J. Comput. Phys., 152:281304, 1999.

[25] F. Otto, P. Penzler, A. Rätz, T. Rump, and A. Voigt. A diffuse interface approximation for step flow in epitaxial growth. Preprint No. 35, caesar, 2003 (submitted).

[26] M. Peterson, C. Ratsch, R. E. Caflisch, and A. Zangwill. Level set approach to reversible epitaxial growth. Phys. Rev. E, 64:061602, 2001.

[27] O. Pierre-Louis. Continuum model for low temperature relaxation of crystal shapes. Phys. Rev. Lett., 87:106104-1-4, 2001.

[28] A. Pimpinelli and J. Villain. Physics of Crystal Growth. Cambridge University Press, Cambridge, 1998.

[29] A. Pimpinelli, J. Villain, D. E. Wolf, J. J. Métois, J. C. Heyraud, I. Elkinani, and G. Uimin. Equilibrium step dynamics on vicinal surfaces. Surface Sci., 295:143, 1993.

[30] P. Politi, G. Grenet, A. Marty, A. Ponchet, and J. Villain. Instabilities in crystal growth by atomic or molecular beams. Physics Reports, 324:271-404, 2000.

[31] P. Politi and J. Villain. Ehrlich-Schwoebel instability in molecular-beam epitaxy: A minimal model. Phys. Rev. B, 54(7):5114-5129, 1996.

[32] C. Ratsch, M. F. Gyure, R. E. Caflisch, F. Gibou, M. Peterson, M. Kang, J. Garcia, and D. D. Vvedensky. Level-set method for island dynamics in epitaxial growth. Phys. Rev. B, 65:195403, 2002.

[33] A. Rätz and A. Voigt. Phase-field models for island dynamics in epitaxial growth. caesar Preprint 33-2003, 2003 (submitted).

[34] A. Schmidt. Computation of three dimensional dendrites with finite elements. $J$. Comput. Phys, 125:293-312, 1996.

[35] A. Schmidt and K. G. Siebert. Albert - software for scientific computations and applications. Acta Math. Univ. Comenianae, 70:105-122, 2001.

[36] R. L. Schwoebel. Step motion on crystal surfaces II. J. Appl. Phys., 40:614-618, 1969. 
[37] R. L. Schwoebel and E. J. Shipsey. Step motion on crystal surfaces. J. Appl. Phys., 37:3682-3686, 1966.

[38] P. Smereka. Semi-implicit level set methods for motion by mean curvature and surface diffusion. Preprint, 2002.

[39] A.-K. Tornberg. Interface Tracking Methods with Application to Multiphase Flows. PhD thesis, NADA, KTH, Stockholm, Sweden, 2000.

[40] J. Y. Tsao. Materials Fundamentals of Molecular Beam Epitaxy. Academic Press, 1993.

[41] J. Villain. Continuum models of crystal growth from atomic beams with and without desorption. J. de Phys. I, 1:19-42, 1991. 